\title{
BOUNDARY HARNACK INEQUALITY FOR MARKOV PROCESSES WITH JUMPS
}

\author{
KRZYSZTOF BOGDAN, TAKASHI KUMAGAI, AND MATEUSZ KWAŚNICKI
}

\begin{abstract}
We prove a boundary Harnack inequality for jump-type Markov processes on metric measure state spaces, under comparability estimates of the jump kernel and Urysohn-type property of the domain of the generator of the process. The result holds for positive harmonic functions in arbitrary open sets. It applies, e.g., to many subordinate Brownian motions, Lévy processes with and without continuous part, stable-like and censored stable processes, jump processes on fractals, and rather general Schrödinger, drift and jump perturbations of such processes.
\end{abstract}

\section{INTRODUCTION}

The boundary Harnack inequality (BHI) is a statement about nonnegative functions which are harmonic on an open set and vanish outside the set near a part of its boundary. BHI asserts that the functions have a common boundary decay rate. The property requires proper assumptions on the set and the underlying Markov process, ones which secure relatively good communication from near the boundary to the center of the set. By this we mean that the process starting near the boundary is going to visit the center of the set at least as likely as to creep far along the boundary before leaving the set.

BHI for harmonic functions of the Laplacian $\Delta$ in Lipschitz domains was proved in 1977-78 by B. Dahlberg, A. Ancona and J.-M. Wu ([4,38,83]), after a pioneering attempt of J. Kemper ([57, 58]). In 1989 R. Bass and K. Burdzy proposed an alternative probabilistic proof based on elementary properties of the Brownian motion ([13]). The resulting 'box method' was then applied to more general domains, including Hölder domains of order $r>1 / 2$, and to more general second order elliptic operators $(14,15)$. BHI trivially fails for disconnected sets, and counterexamples for Hölder domains with $r<1 / 2$ are given in [15]. In 2001-09, H. Aikawa studied BHI for classical harmonic functions in connection to the Carleson estimate and under exterior capacity conditions ([1] 3] ).

Moving on to nonlocal operators and jump-type Markov processes, in 1997 K. Bogdan proved BHI for the fractional Laplacian $\Delta^{\alpha / 2}$ (and the isotropic $\alpha$ stable Lévy process) for $0<\alpha<2$ and Lipschitz sets ([19]). In 1999 R. Song and

Received by the editors July 16, 2012 and, in revised form, March 2, 2013 and March 9, 2013. 2010 Mathematics Subject Classification. Primary 60J50; Secondary 60J75, 31B05.

Key words and phrases. Boundary Harnack inequality, jump Markov process.

The first author was supported in part by grant N N201 397137.

The second author was supported by the Grant-in-Aid for Challenging Exploratory Research 24654033

The third author was supported by the Foundation for Polish Science and by the Polish National Science Centre grant no. 2011/03/D/ST1/00311. 
J. -M. Wu extended the results to the so-called fat sets ([75), and in $2007 \mathrm{~K}$. Bogdan, T. Kulczycki and M. Kwaśnicki proved BHI for $\Delta^{\alpha / 2}$ in arbitrary, in particular disconnected, open sets ([26]). In 2008 P. Kim, R. Song and Z. Vondraček proved BHI for subordinate Brownian motions in fat sets (63]) and in 2011 extended it to a general class of isotropic Lévy processes and arbitrary domains (65]). Quite recently, BHI for $\Delta+\Delta^{\alpha / 2}$ was established by Z.-Q. Chen, P. Kim, R. Song and Z. Vondraček [32. We would also like to mention BHI for censored processes [21,44] by K. Bogdan, K. Burdzy, Z.-Q. Chen and Q. Guan, and fractal jump processes [55,77] by K. Kaleta, M. Kwaśnicki and A. Stós.

Generally speaking, BHI is more a topological issue for diffusion processes and more a measure-theoretic issue for jump-type Markov processes, which may transport from near the boundary to the center of the set by direct jumps. However, [19,26] show in a special setting that such jumps determine the asymptotics of harmonic functions only at those boundary points where the set is rather thin, while at other boundary points the main contribution to the asymptotics comes from gradual 'excursions' away from the boundary.

We recall that BHI in particular applies to and may yield an approximate factorization of the Green function. This line of research was completed for Lipschitz domains in 2000 by K. Bogdan ([20]) for $\Delta$ and in 2002 by T. Jakubowski ([52]) for $\Delta^{\alpha / 2}$. It is now a well-established technique (47]) and extensions were proved, e.g., for subordinate Brownian motions by P. Kim, R. Song and Z. Vondraček ([66]). We should note that so far the technique is typically restricted to Lipschitz or fat sets. Furthermore, for smooth sets, e.g. $C^{1,1}$ sets, the approximate factorization is usually more explicit. This is so because for smooth sets the decay rate in BHI can often be explicitly expressed in terms of the distance to the boundary of the set. The first complete results in this direction were given for $\Delta$ in 1986 by Z. Zhao (84]) and for $\Delta^{\alpha / 2}$ in 1997 by T. Kulczycki ([67]) and in 1998 by Z.-Q. Chen and R. Song ([36]). The estimates are now extended to subordinate Brownian motions, and the renewal function of the subordinator is used in the corresponding formulations ([6] ). Accordingly, the Green function of smooth sets enjoys approximate factorization for rather general isotropic Lévy processes $([29,66])$. We expect further progress in this direction with applications to perturbation theory via the so-called 3G theorems, and to nonlinear partial differential equations $([25,47,70])$. We should also mention estimates and approximate factorization of the Dirichlet heat kernels, which are intensively studied at present. The estimates depend on BHI ([24]) and reflect the fundamental decay rate in BHI ([31,46]).

BHI tends to self-improve and may lead to the existence of the boundary limits of ratios of nonnegative harmonic functions, thanks to oscillation reduction (13. 19, 26, 54 ). The oscillation reduction technique is rather straightforward for local operators. It is more challenging for nonlocal operators, as it involves subtraction of harmonic functions, which destroys global nonnegativity. The technique requires a certain scale invariance, or uniformity of BHI, and works, e.g., for $\Delta$ in Lipschitz domains ([13]) and for $\Delta^{\alpha / 2}$ in arbitrary domains $([26])$. We should remark that Hölder continuity of harmonic functions is a similar phenomenon, related to the usual Harnack inequality, and that BHI extends the usual Harnack inequality if, e.g., constant functions are harmonic. Hölder continuity of harmonic functions is crucial in the theory of partial differential equations [6, 16, and the existence of 
limits of ratios of nonnegative harmonic functions leads to the construction of the Martin kernel and to the representation of nonnegative harmonic functions $([\underline{5}, 26])$.

The above summary indicates further directions of research resulting from our development. The main goal of this article is to study the following boundary Harnack inequality. In Section 2 we specify notation and assumptions which validate the estimate.

(BHI) Let $x_{0} \in \mathfrak{X}, 0<r<R<R_{0}$, and let $D \subseteq B\left(x_{0}, R\right)$ be open. Suppose that nonnegative functions $f, g$ on $\mathfrak{X}$ are regular harmonic in $D$ with respect to the process $X_{t}$, and vanish in $B\left(x_{0}, R\right) \backslash D$. There is $c_{\text {[1.1 }}=c_{\text {[1.1 }}\left(x_{0}, r, R\right)$ such that

$$
f(x) g(y) \leq c_{1.1]} f(y) g(x), \quad x, y \in B\left(x_{0}, r\right) .
$$

Here $X_{t}$ is a Hunt process, having a metric measure space $\mathfrak{X}$ as the state space, and $R_{0} \in(0, \infty]$ is a localization radius (discussed in Section 2). Also, a nonnegative function $f$ is said to be regular harmonic in $D$ with respect to $X_{t}$ if

$$
f(x)=\mathbf{E}_{x} f\left(X_{\tau_{D}}\right), \quad x \in D,
$$

where $\tau_{D}$ is the time of the first exit of $X_{t}$ from $D$. To facilitate cross-referencing, in (1.1) and later on we let $c_{(i)}$ denote the constant in the displayed formula $(i)$. By $c$ or $c_{i}$ we denote secondary (temporary) constants in a lemma or a section, and $c=c(a, \ldots, z)$, or simply $c(a, \ldots, z)$, means a constant $c$ that may be so chosen to depend only on $a, \ldots, z$. Throughout the article, all constants are positive.

The present work started with an attempt to obtain bounded kernels which reproduce harmonic functions. We were motivated by the so-called regularization of the Poisson kernel for $\Delta^{\alpha / 2}([22]$, [26, Lemma 6]), which is crucial for the Carleson estimate and BHI for $\Delta^{\alpha / 2}$. In the present paper we construct kernels obtained by gradually stopping the Markov process with a specific multiplicative functional before the process approaches the boundary. The construction is the main technical ingredient of our work, and is presented in Section 4. The argument is intrinsically probabilistic and relies on delicate analysis on the path space. At the beginning of Section 4 the reader will also find a short informal presentation of the construction. Section 2 gives assumptions and auxiliary results. The boundary Harnack inequality (Theorem 3.5), and the so-called local supremum estimate (Theorem 3.4) are presented in Section 3, but the proof of Theorem 3.4 is deferred to Section 4 . In Section 5 we verify in various settings the scale-invariance of BHI, discuss the relevance of our main assumptions from Section 2, and present many applications, including subordinate Brownian motions, Lévy processes with or without continuous part, stable-like and censored processes, Schrödinger, gradient and jump perturbations, processes on fractals and more.

\section{Assumptions And Preliminaries}

Let $(\mathfrak{X}, d, m)$ be a metric measure space such that all bounded closed sets are compact and $m$ has full support. Let $B(x, r)=\{y \in \mathfrak{X}: d(x, y)<r\}$, where $x \in \mathfrak{X}$ and $r>0$. All sets, functions and measures considered in this paper are Borel. Let $R_{0} \in(0, \infty]$ (the localization radius) be such that $\mathfrak{X} \backslash B(x, 2 r) \neq \varnothing$ for all $x \in \mathfrak{X}$ and all $r<R_{0}$. Let $\mathfrak{X} \cup\{\partial\}$ be the one-point compactification of $\mathfrak{X}$ (if $\mathfrak{X}$ is compact, then we add $\partial$ as an isolated point). Without much mention we extend functions $f$ on $\mathfrak{X}$ to $\mathfrak{X} \cup\{\partial\}$ by letting $f(\partial)=0$. In particular, we 
write $f \in C_{0}(\mathfrak{X})$ if $f$ is a continuous real-valued function on $\mathfrak{X} \cup\{\partial\}$ and $f(\partial)=0$. If furthermore $f$ has compact support in $\mathfrak{X}$, then we write $f \in C_{c}(\mathfrak{X})$. For a kernel $k(x, d y)$ on $\mathfrak{X}([39])$ we let $k f(x)=\int f(y) k(x, d y)$, provided the integral makes sense, i.e., $f$ is (measurable and) either nonnegative or absolutely integrable. Similarly, for a kernel density function $k(x, y) \geq 0$, we let $k(x, E)=\int_{E} k(x, y) m(d y)$ and $k(E, y)=\int_{E} k(x, y) m(d x)$ for $E \subseteq \mathfrak{X}$.

Let $\left(X_{t}, \zeta, \mathcal{M}_{t}, \mathbf{P}_{x}\right)$ be a Hunt process with state space $\mathfrak{X}$ (see, e.g., [18, I.9] or [40, 3.23]). Here $X_{t}$ are the random variables, $\mathcal{M}_{t}$ is the usual right-continuous filtration, $\mathbf{P}_{x}$ is the distribution of the process starting from $x \in \mathfrak{X}$, and $\mathbf{E}_{x}$ is the corresponding expectation. The random variable $\zeta \in(0, \infty]$ is the lifetime of $X_{t}$, so that $X_{t}=\partial$ for $t \geq \zeta$. This should be kept in mind when interpreting (1.2) above, (2.1) below, etc. The transition operators of $X_{t}$ are defined by

$$
T_{t} f(x)=\mathbf{E}_{x} f\left(X_{t}\right), \quad t \geq 0, x \in \mathfrak{X},
$$

whenever the expectation makes sense. We assume that the semigroup $T_{t}$ is Feller and strong Feller; i.e., for $t>0, T_{t}$ maps bounded functions into continuous ones and $C_{0}(\mathfrak{X})$ into $C_{0}(\mathfrak{X})$. The Feller generator $\mathcal{A}$ of $X_{t}$ is defined on the set $\mathcal{D}(\mathcal{A})$ of all those $f \in C_{0}(\mathfrak{X})$ for which the limit

$$
\mathcal{A} f(x)=\lim _{t \searrow 0} \frac{T_{t} f(x)-f(x)}{t}
$$

exists uniformly in $x \in \mathfrak{X}$. The $\alpha$-potential operator,

$$
\mathcal{U}_{\alpha} f(x)=\mathbf{E}_{x} \int_{0}^{\infty} f\left(X_{t}\right) e^{-\alpha t} d t=\int_{0}^{\infty} e^{-\alpha t} T_{t} f(x) d t, \quad \alpha \geq 0, x \in \mathfrak{X},
$$

is defined whenever the expectation makes sense. We let $\mathcal{U}=\mathcal{U}_{0}$, the potential operator. The kernels of $T_{t}, \mathcal{U}_{\alpha}$ and $\mathcal{U}$ are denoted by $T_{t}(x, d y), \mathcal{U}_{\alpha}(x, d y)$ and $\mathcal{U}(x, d y)$, respectively.

Recall that a function $f \geq 0$ is called $\alpha$-excessive (with respect to $T_{t}$ ) if for all $x \in \mathfrak{X}, e^{-\alpha t} T_{t} f(x) \leq f(x)$ for $t>0$, and $e^{-\alpha t} T_{t} f(x) \rightarrow f(x)$ as $t \rightarrow 0^{+}$. When $\alpha=0$, we simply say that $f$ is excessive.

We enforce a number of conditions, namely Assumptions $\mathrm{A}, \mathrm{B}, \mathrm{C}$ and D below. We start with a duality assumption, which builds on our discussion of $X_{t}$.

Assumption A. There are Hunt processes $X_{t}$ and $\hat{X}_{t}$ which are dual with respect to the measure $m$ (see [18, VI.1] or [37, 13.1]). The transition semigroups of $X_{t}$ and $\hat{X}_{t}$ are both Feller and strong Feller. Every semipolar set of $X_{t}$ is polar.

In what follows, objects pertaining to $\hat{X}_{t}$ are distinguished in notation from those for $X_{t}$ by adding a hat over the corresponding symbol. For example, $\hat{T}_{t}$ and $\hat{\mathcal{U}}_{\alpha}$ denote the transition and $\alpha$-potential operators of $\hat{X}_{t}$. The first sentence of Assumption $\mathrm{A}$ means that for all $\alpha>0$, there are functions $\mathcal{U}_{\alpha}(x, y)=\hat{\mathcal{U}}_{\alpha}(y, x)$ such that

$$
\mathcal{U}_{\alpha} f(x)=\int_{\mathfrak{X}} \mathcal{U}_{\alpha}(x, y) f(y) m(d y), \quad \hat{\mathcal{U}}_{\alpha} f(x)=\int_{\mathfrak{X}} \hat{\mathcal{U}}_{\alpha}(x, y) f(y) m(d y)
$$

for all $f \geq 0$ and $x \in \mathfrak{X}$, and such that $x \mapsto \mathcal{U}_{\alpha}(x, y)$ is $\alpha$-excessive with respect to $T_{t}$, and $y \mapsto \mathcal{U}_{\alpha}(x, y)$ is $\alpha$-excessive with respect to $\hat{T}_{t}$ (that is, $\alpha$-co-excessive). The $\alpha$-potential kernel $\mathcal{U}_{\alpha}(x, y)$ is unique (see [37, Theorem 13.2] or the remarks after [18, Proposition VI.1.3]). 
The condition in Assumption $\mathrm{A}$ that semipolar sets are polar is also known as Hunt's hypothesis $(\mathrm{H})$. Most notably, it implies that the process $X_{t}$ never hits irregular points; see, e.g., [18, I.11 and II.3] or [37, Chapter 3]. The $\alpha$-potential kernel is nonincreasing in $\alpha>0$, and hence the potential kernel $\mathcal{U}(x, y)=\lim _{t \rightarrow 0^{+}} \mathcal{U}_{\alpha}(x, y) \in$ $[0, \infty]$ is well-defined. $\hat{X}_{t}$,

We consider an open set $D \subset \mathfrak{X}$ and the time of the first exit from $D$ for $X_{t}$ and

$$
\tau_{D}=\inf \left\{t \geq 0: X_{t} \notin D\right\} \quad \text { and } \quad \hat{\tau}_{D}=\inf \left\{t \geq 0: \hat{X}_{t} \notin D\right\} .
$$

We define the processes killed at $\tau_{D}$,

$$
X_{t}^{D}=\left\{\begin{array}{ll}
X_{t}, & \text { if } t<\tau_{D}, \\
\partial, & \text { if } t \geq \tau_{D},
\end{array} \quad \text { and } \quad \hat{X}_{t}^{D}= \begin{cases}\hat{X}_{t}, & \text { if } t<\hat{\tau}_{D}, \\
\partial, & \text { if } t \geq \hat{\tau}_{D}\end{cases}\right.
$$

We let $T_{t}^{D}(x, d y)$ and $\hat{T}_{t}^{D}(x, d y)$ be their transition kernels. By [37, Remark 13.26], $X_{t}^{D}$ and $\hat{X}_{t}^{D}$ are dual processes with state space $D$. Indeed, for each $x \in D, \mathbf{P}_{x}$-a.s. the process $X_{t}$ only hits regular points of $\mathfrak{X} \backslash D$ when it exits $D$. In the nomenclature of [37, 13.6], this means that the left-entrance time and the hitting time of $\mathfrak{X} \backslash D$ are equal $\mathbf{P}^{x}$-a.s. for every $x \in D$. In particular, the potential kernel $G_{D}(x, y)$ of $X_{t}^{D}$ exists and is unique, although in general it may be infinite ([18, pp. 256-257]). $G_{D}(x, y)$ is called the Green function for $X_{t}$ on $D$, and it defines the Green operator $G_{D}$,

$$
G_{D} f(x)=\int_{\mathfrak{X}} f(y) G_{D}(x, y) m(d y)=\mathbf{E}_{x} \int_{0}^{\tau_{D}} f\left(X_{t}\right) d t, \quad x \in \mathfrak{X}, f \geq 0 .
$$

Note that $\mathcal{U}(x, y)=G_{\mathfrak{X}}(x, y)$. When $X_{t}$ is symmetric (self-dual) with respect to $m$, then Assumption $\mathrm{A}$ is equivalent to the existence of the $\alpha$-potential kernel $\mathcal{U}_{\alpha}(x, y)$ for $X_{t}$, since then Hunt's hypothesis $(\mathrm{H})$ is automatically satisfied; see [37.

The following Urysohn regularity hypothesis plays a crucial role in our paper, providing enough 'smooth' functions on $\mathfrak{X}$ to approximate indicator functions of compact sets.

Assumption B. There is a linear subspace $\mathcal{D}$ of $\mathcal{D}(\mathcal{A}) \cap \mathcal{D}(\hat{\mathcal{A}})$ satisfying the following condition. If $K$ is compact, $D$ is open, and $K \subseteq D \subseteq \mathfrak{X}$, then there is $f \in \mathcal{D}$ such that $f(x)=1$ for $x \in K, f(x)=0$ for $x \in \mathfrak{X} \backslash D, 0 \leq f(x) \leq 1$ for $x \in \mathfrak{X}$, and the boundary of the set $\{x: f(x)>0\}$ has measure $m$ zero. We let

$$
\varrho(K, D)=\inf _{f} \sup _{x \in \mathfrak{X}} \max (\mathcal{A} f(x), \hat{\mathcal{A}} f(x)),
$$

where the infimum is taken over all such functions $f$.

Thus, nonnegative functions in $\mathcal{D}(\mathcal{A}) \cap \mathcal{D}(\hat{\mathcal{A}})$ separate the compact set $K$ from the closed set $\mathfrak{X} \backslash D$ : there is a Urysohn (bump) function for $K$ and $\mathfrak{X} \backslash D$ in the domains. Since the supremum in (2.2) is finite for any $f \in \mathcal{D}$ and the infimum is taken over a nonempty set, $\varrho(K, D)$ is always finite.

Note that constant functions are not in $\mathcal{D}(\mathcal{A})$ or $\mathcal{D}(\hat{\mathcal{A}})$ unless $\mathfrak{X}$ is compact. In the Euclidean case $\mathfrak{X}=\mathbf{R}^{d}, \mathcal{D}$ can often be taken as the class $C_{c}^{\infty}\left(\mathbf{R}^{d}\right)$ of compactly supported smooth functions. The existence of $\mathcal{D}$ is problematic if $\mathfrak{X}$ is more general. However, for the Sierpiński gasket and some other self-similar (p.c.f.) fractals, $\mathcal{D}$ can be constructed by using the concept of splines on fractals $([55,78])$. Also, a class of smooth indicator functions was recently constructed in [1] for heat 
kernels satisfying upper sub-Gaussian estimates on $\mathfrak{X}$. Further discussion is given in Section 5 and Appendix $\mathrm{A}$. Here we note that Assumption B implies that the jumps of $X_{t}$ are subject to the following identity, which we call the Lévy system formula for $X_{t}$ :

$$
\mathbf{E}_{x} \sum_{s \in[0, t]} f\left(s, X_{s-}, X_{s}\right)=\mathbf{E}_{x} \int_{0}^{t} \int_{\mathfrak{X}} f\left(s, X_{s-}, z\right) \nu\left(X_{s-}, d z\right) d s .
$$

Here $f:[0, \infty) \times \mathfrak{X} \times \mathfrak{X} \rightarrow[0, \infty], f(x, x)=0$ for all $x \in \mathfrak{X}$, and $\nu$ is a kernel on $\mathfrak{X}$ (satisfying $\nu(x,\{x\})=0$ for all $x \in \mathfrak{X}$ ), called the Lévy kernel of $X_{t}$; see [17,74,80]. For more general Markov processes, $d s$ in (2.3) is superseded by the differential of a perfect, continuous additive functional, and (2.3) defines $\nu(x, \cdot)$ only up to a set of zero potential, that is, for $m$-almost every $x \in \mathfrak{X}$. By inspecting the construction in [17, 74, and using Assumption B, one proves in a similar way as in [12, Section 5] that the Lévy kernel $\nu$ satisfies

$$
\nu f(x)=\lim _{t \searrow 0} \frac{T_{t} f(x)}{t}, \quad f \in C_{c}(\mathfrak{X}), x \in \mathfrak{X} \backslash \operatorname{supp} f .
$$

This formula, as opposed to (2.3), defines $\nu(x, d y)$ for all $x \in \mathfrak{X}$. With only one exception, to be discussed momentarily, we use (2.4) and not (2.3); hence we take (2.4) as the definition of $\nu$. It is easy to see that (2.4) indeed defines $\nu(x, d y)$ : if $f \in \mathcal{D}(\mathcal{A})$ and $x \in \mathfrak{X} \backslash \operatorname{supp} f$, then $\nu f(x)=\mathcal{A} f(x)$. By Assumption B the mapping $f \mapsto \nu f(x)$ is a densely defined, nonnegative linear functional on $C_{c}(\mathfrak{X} \backslash\{x\})$; hence it corresponds to a nonnegative Radon measure $\nu(x, d y)$ on $\mathfrak{X} \backslash\{x\}$. As usual, we let $\nu(x,\{x\})=0$. The Lévy kernel $\hat{\nu}(y, d x)$ for $\hat{X}_{t}$ is defined in a similar manner. By duality, $\nu(x, d y) m(d x)=\hat{\nu}(y, d x) m(d y)$.

As an application of (2.3) we consider the martingale

$$
t \mapsto \sum_{s \in[0, t]} f\left(s, X_{s-}, X_{s}\right)-\int_{0}^{t} \int_{\mathfrak{X}} f\left(s, X_{s-}, z\right) \nu\left(X_{s-}, d z\right) d s,
$$

where $f(s, y, z)=\mathbf{1}_{A}(s) \mathbf{1}_{E}(y) \mathbf{1}_{F}(z)$. We stop the martingale at $\tau_{D}$ and we see that

$$
\mathbf{P}_{x}\left(\tau_{D} \in d t, X_{\tau_{D}-} \in d y, X_{\tau_{D}} \in d z\right)=d t T_{t}^{D}(x, d y) \nu(y, d z),
$$

on $(0, \infty) \times D \times(\mathfrak{X} \backslash D)$. A similar result was first proved in [51]. For this reason we refer to (2.5) as the Ikeda-Watanabe formula (see also (2.12) and (2.6) below). Integrating (2.5) against $d t$ and $d y$ we obtain

$$
\mathbf{P}_{x}\left(X_{\tau_{D}-} \neq X_{\tau_{D}}, X_{\tau_{D}} \in E\right)=\int_{D} G_{D}(x, d y) \nu(y, E), \quad x \in D, E \subset \mathfrak{X} \backslash D .
$$

For $x_{0} \in \mathfrak{X}$ and $0<r<R$, we consider the open and closed balls $B\left(x_{0}, r\right)=$ $\left\{x \in \mathfrak{X}: d\left(x_{0}, x\right)<r\right\}$ and $\bar{B}\left(x_{0}, r\right)=\left\{x \in \mathfrak{X}: d\left(x_{0}, x\right) \leq r\right\}$, and the annular regions $A\left(x_{0}, r, R\right)=\left\{x \in \mathfrak{X}: r<d\left(x_{0}, x\right)<R\right\}$ and $\bar{A}\left(x_{0}, r, R\right)=\{x \in \mathfrak{X}: r \leq$ $\left.d\left(x_{0}, x\right) \leq R\right\}$. Note that $\overline{B\left(x_{0}, r\right)}$, the closure of $B\left(x_{0}, r\right)$, may be a proper subset of $\bar{B}\left(x_{0}, r\right)$.

Recall that $R_{0}$ denotes the localization radius of $\mathfrak{X}$. The following assumption is our main condition for the boundary Harnack inequality. It asserts a relative constancy of the density of the Lévy kernel. This is a natural condition, as seen in Example 5.14. 
Assumption C. The Lévy kernels of the processes $X_{t}$ and $\hat{X}_{t}$ have the form $\nu(x, y) m(d y)$ and $\hat{\nu}(x, y) m(d y)$ respectively, where $\nu(x, y)=\hat{\nu}(y, x)>0$ for all $x, y \in \mathfrak{X}, x \neq y$. For every $x_{0} \in \mathfrak{X}, 0<r<R<R_{0}, x \in B\left(x_{0}, r\right)$ and $y \in \mathfrak{X} \backslash B\left(x_{0}, R\right)$,

$$
\begin{aligned}
& c_{\sqrt{2.7}}^{-1} \nu\left(x_{0}, y\right) \leq \nu(x, y) \leq c_{(2.7)} \nu\left(x_{0}, y\right), \\
& c_{[2.7]}^{-1} \hat{\nu}\left(x_{0}, y\right) \leq \hat{\nu}(x, y) \leq c_{[2.7} \hat{\nu}\left(x_{0}, y\right),
\end{aligned}
$$

with $c_{[2.7}=c_{[2.7)}\left(x_{0}, r, R\right)$.

It follows directly from Assumption $\mathrm{C}$ that for $x_{0} \in \mathfrak{X}$ and $0<r<R$,

$$
c_{[2.8]}\left(x_{0}, r, R\right)=\inf _{y \in \bar{A}\left(x_{0}, r, R\right)} \min \left(\nu\left(x_{0}, y\right), \hat{\nu}\left(x_{0}, y\right)\right)>0,
$$

where $\bar{A}\left(x_{0}, r, R\right)=\left\{x \in \mathfrak{X}: r \leq d\left(x_{0}, x\right) \leq R\right\}$. (Here we do not require that $R<$ $R_{0}$.) Indeed, we may cover $\bar{A}\left(x_{0}, r, R\right)$ by a finite family of balls $B\left(y_{i}, r / 2\right)$, where $y_{i} \in \bar{A}\left(x_{0}, r, R\right)$. For $y \in B\left(y_{i}, r / 2\right), \nu\left(x_{0}, y\right)$ is comparable with $\nu\left(x_{0}, y_{i}\right)$, and $\hat{\nu}\left(x_{0}, y\right)$ is comparable with $\hat{\nu}\left(x_{0}, y_{i}\right)$.

Proposition 2.1. If $x_{0} \in \mathfrak{X}$ and $0<r<R_{0}$, then

$$
c_{[2.9)}\left(x_{0}, r\right)=\sup _{x \in B\left(x_{0}, r\right)} \max \left(\mathbf{E}_{x} \tau_{B\left(x_{0}, r\right)}, \hat{\mathbf{E}}_{x} \hat{\tau}_{B\left(x_{0}, r\right)}\right)<\infty .
$$

Proof. Let $B=B\left(x_{0}, r\right), R \in\left(r, R_{0}\right), x, y \in B$ and $F(t)=\mathbf{P}_{x}\left(\tau_{B}>t\right)$. By the definition of $R_{0}, m\left(\mathfrak{X} \backslash B\left(x_{0}, R\right)\right)>0$. This and (2.7) yield $\nu(y, \mathfrak{X} \backslash B) \geq$ $\nu\left(y, \mathfrak{X} \backslash B\left(x_{0}, R\right)\right) \geq\left(c_{[2.7]}\left(x_{0}, r, R\right)\right)^{-1} \nu\left(x_{0}, \mathfrak{X} \backslash B\left(x_{0}, R\right)\right)=c . \quad$ By the IkedaWatanabe formula (2.5),

$$
\begin{aligned}
-F^{\prime}(t) & =\frac{\mathbf{P}_{x}\left(\tau_{B} \in d t\right)}{d t} \geq \frac{\mathbf{P}_{x}\left(\tau_{B} \in d t, X_{\tau_{B}-} \neq X_{\tau_{B}}, X_{\tau_{B}} \in \mathfrak{X} \backslash B\right)}{d t} \\
& =\int_{\mathfrak{X}} \nu(y, \mathfrak{X} \backslash B) T_{t}^{B}(x, d y) \geq c \int_{\mathfrak{X}} T_{t}^{B}(x, d y)=c F(t) .
\end{aligned}
$$

Hence $\mathbf{P}_{x}\left(\tau_{B}>t\right) \leq e^{-c t}$. It follows that $\mathbf{E}_{x} \tau_{B} \leq 1 / c$. Considering an analogous argument for $\hat{\mathbf{E}}_{x} \hat{\tau}_{B}$, we see that we may take

$$
c_{[\mathbf{2 . 9}}\left(x_{0}, r\right)=\inf _{R \in\left(r, R_{0}\right)} \max \left(\frac{c_{[2.7]}\left(x_{0}, r, R\right)}{\nu\left(x_{0}, \mathfrak{X} \backslash B\left(x_{0}, R\right)\right)}, \frac{c_{[2.7]}\left(x_{0}, r, R\right)}{\hat{\nu}\left(x_{0}, \mathfrak{X} \backslash B\left(x_{0}, R\right)\right)}\right) .
$$

In particular, if $0<R<R_{0}$ and $D \subseteq B\left(x_{0}, R\right)$, then the Green function $G_{D}(x, y)$ exists (see the discussion following Assumption $\underline{\mathrm{A}}$ ), and for each $x \in \mathfrak{X}$ it is finite for all $y$ in $\mathfrak{X}$ less a polar set. We need to assume slightly more. The following condition may be viewed as a weak version of Harnack's inequality.

Assumption D. If $x_{0} \in \mathfrak{X}, 0<r<p<R<R_{0}$ and $B=B\left(x_{0}, R\right)$, then

$$
c_{[2.10)}\left(x_{0}, r, p, R\right)=\sup _{x \in B\left(x_{0}, r\right)} \sup _{y \in \mathfrak{X} \backslash B\left(x_{0}, p\right)} \max \left(G_{B}(x, y), \hat{G}_{B}(x, y)\right)<\infty .
$$

Assumptions $\mathrm{A}, \mathrm{B}, \mathrm{C}$ and $\mathrm{D}$ are tacitly assumed throughout the entire paper. We recall them explicitly only in the statements of $\mathrm{BHI}$ and local maximum estimate.

When saying that a statement holds for almost every point of $\mathfrak{X}$, we refer to the measure $m$. The following technical result is a simple generalization of [18, Proposition II.3.2]. 
Proposition 2.2. Suppose that $Y_{t}$ is a standard Markov process such that for every $x \in \mathfrak{X}$ and $\alpha>0$, the $\alpha$-potential kernel $\mathcal{V}_{\alpha}(x, d y)$ of $Y_{t}$ is absolutely continuous with respect to $m(d y)$. Suppose that function $f$ is excessive for the transition semigroup of $Y_{t}$, and $f$ is not identically infinite. If function $g$ is continuous and $f(x) \leq g(x)$ for almost every $x \in B\left(x_{0}, r\right)$, then $f(x) \leq g(x)$ for every $x \in B\left(x_{0}, r\right)$.

Proof. Let $A=\left\{x \in B\left(x_{0}, r\right): f(x)>g(x)\right\}$. Then $m(A)=0$, so that $A$ is of zero potential for $Y$. Hence $B\left(x_{0}, r\right) \backslash A$ is finely dense in $B\left(x_{0}, r\right)$. Since $f-g$ is finely continuous, we have $f(x) \leq g(x)$ for all $x \in B\left(x_{0}, r\right)$, as desired. (See e.g. [18, 37] for the notion of fine topology and fine continuity of excessive functions.)

If $X_{t}$ is transient, (2.10) often holds even when $G_{B}$ is replaced by $G_{\mathfrak{X}}=\mathcal{U}$. In the recurrent case, we can use estimates of $\mathcal{U}_{\alpha}$, as follows.

Proposition 2.3. If $x_{0} \in \mathfrak{X}, 0<r<p<R<R_{0}, \alpha>0$,

$$
c_{1}\left(x_{0}, r, p, \alpha\right)=\sup _{x \in B\left(x_{0}, r\right)} \sup _{y \in \mathfrak{X} \backslash B\left(x_{0}, p\right)} \max \left(\mathcal{U}_{\alpha}(x, y), \hat{\mathcal{U}}_{\alpha}(x, y)\right)<\infty,
$$

and $T_{t}(x, d y) \leq c_{2}(t) m(d y)$ for all $x, y \in \mathfrak{X}, t>0$, then in (2.10) we may let

$$
c_{[2.10)}\left(x_{0}, r, p, R\right)=\inf _{\alpha, t>0}\left(e^{\alpha t} c_{1}\left(x_{0}, r, p, \alpha\right)+c_{2}(t) c_{[2.9)}\left(x_{0}, R\right)\right) \text {. }
$$

Proof. Denote $B=B\left(x_{0}, R\right)$. If $x \in B\left(x_{0}, r\right), t_{0}>0$ and $E \subseteq B \backslash B\left(x_{0}, p\right)$, then

$$
\begin{aligned}
G_{B} \mathbf{1}_{E}(x) & =\int_{0}^{\infty} T_{t}^{B} \mathbf{1}_{E}(x) d t \\
& \leq e^{\alpha t_{0}} \int_{0}^{t_{0}} e^{-\alpha t} T_{t}^{B} \mathbf{1}_{E}(x) d t+\int_{0}^{\infty} T_{s}^{B}\left(T_{t_{0}}^{B} \mathbf{1}_{E}\right)(x) d s \\
& \leq e^{\alpha t_{0}} \int_{0}^{\infty} e^{-\alpha t} T_{t} \mathbf{1}_{E}(x) d t+\left(\sup _{y \in B} T_{t_{0}}^{B} \mathbf{1}_{E}(y)\right) \int_{0}^{\infty} T_{s}^{B} \mathbf{1}(y) d s \\
& \leq e^{\alpha t_{0}} \mathcal{U}_{\alpha} \mathbf{1}_{E}(x)+\left(\sup _{y \in B} T_{t_{0}} \mathbf{1}_{E}(y)\right) G_{B} \mathbf{1}(x) \\
& \leq\left(e^{\alpha t_{0}} c_{1}+c_{2} G_{B} \mathbf{1}(x)\right)|E|,
\end{aligned}
$$

where $c_{1}=c_{1}\left(x_{0}, r, p, \alpha\right)$ and $c_{2}=c_{2}\left(t_{0}\right)$. If $y \in B \backslash B\left(x_{0}, p\right)$, then by Proposition [2.2. $G_{B}(x, y) \leq e^{\alpha t_{0}} c_{1}+c_{2} G_{B} \mathbf{1}(x)$. By Proposition 2.1], $G_{B} \mathbf{1}(x)=\mathbf{E}_{x} \tau_{B} \leq$ $c_{\text {(2.9) }}\left(x_{0}, R\right)$. The estimate of $\hat{G}_{B}(x, y)$ is similar.

We use the standard notation $\mathbf{E}_{x}(Z ; E)=\mathbf{E}_{x}\left(Z \mathbf{1}_{E}\right)$. Recall that all functions $f$ on $\mathfrak{X}$ are automatically extended to $\mathfrak{X} \cup\{\partial\}$ by letting $f(\partial)=0$. In particular, we understand that $\mathcal{A} f(\partial)=0$ for all $f \in \mathcal{D}(\mathcal{A})$, and $\mathbf{E}_{x} \mathcal{A} f\left(X_{\tau}\right)=\mathbf{E}_{x}\left(\mathcal{A} f\left(X_{\tau}\right) ; \tau<\zeta\right)$.

The following formula obtained by Dynkin (see [40, formula (5.8)]) plays an important role. If $\tau$ is a Markov time, $\mathbf{E}_{x} \tau<\infty$ and $f \in \mathcal{D}(\mathcal{A})$, then

$$
\mathbf{E}_{x} f\left(X_{\tau}\right)=f(x)+\mathbf{E}_{x} \int_{0}^{\tau} \mathcal{A} f\left(X_{t}\right) d t, \quad x \in \mathfrak{X} .
$$

If $D \subseteq B\left(x_{0}, R_{0}\right), f \in \mathcal{D}(\mathcal{A})$ is supported in $\mathfrak{X} \backslash D$ and $X_{t} \in D \mathbf{P}_{y}$-a.s. for $t<\tau$ and $x \in \mathfrak{X}$, then

$$
\begin{aligned}
\mathbf{E}_{x} f\left(X_{\tau}\right) & =\mathbf{E}_{x} \int_{0}^{\tau}\left(\int_{\mathfrak{X}} \nu\left(X_{t}, y\right) f(y) m(d y)\right) d t \\
& =\int_{\mathfrak{X}} \mathbf{E}_{x}\left(\int_{0}^{\tau} \nu\left(X_{t}, y\right) d t\right) f(y) m(d y) .
\end{aligned}
$$


We note that (2.12) extends to nonnegative functions $f$ on $\mathfrak{X}$ which vanish on $\bar{D}$. Indeed, both sides of (2.12) define nonnegative functionals of $f \in C_{0}(\mathfrak{X} \backslash \bar{D})$, and hence also nonnegative Radon measures on $\mathfrak{X} \backslash \bar{D}$. By (2.12), the two functionals coincide on $\mathcal{D} \cap C_{0}(\mathfrak{X} \backslash \bar{D})$, and this set is dense in $C_{0}(\mathfrak{X} \backslash \bar{D})$ by the Urysohn regularity hypothesis. This proves that the corresponding measures are equal. We also note that one cannot in general relax the condition that $f=0$ on $\bar{D}$. Indeed, even if $m(\partial D)=0, X_{\tau}$ may hit $\partial D$ with positive probability.

Recall that a function $f \geq 0$ on $\mathfrak{X}$ is called regular harmonic in an open set $D \subseteq \mathfrak{X}$ if $f(x)=\mathbf{E}_{x} f\left(X\left(\tau_{D}\right)\right)$ for all $x \in \mathfrak{X}$. Here a typical example is $x \mapsto \mathbf{E}_{x} \int_{0}^{\infty} g\left(X_{t}\right) \bar{d} t$ if $g \geq 0$ vanishes on $D$. By the strong Markov property we then have $f(x)=\mathbf{E}_{x} f\left(X_{\tau}\right)$ for all stopping times $\tau \leq \tau_{D}$. Accordingly, we call $f \geq 0$ regular subharmonic in $D$ (for $X_{t}$ ), if $f(x) \leq \mathbf{E}_{x} f\left(X_{\tau}\right)$ for all stopping times $\tau \leq \tau_{D}$ and $x \in \mathfrak{X}$. Here a typical example is a regular harmonic function raised to a power $p \geq 1$. We like to recall that $f \geq 0$ is called harmonic in $D$, if $f(x)=\mathbf{E}_{x} f\left(X\left(\tau_{U}\right)\right)$ for all open and bounded $U$ such that $\bar{U} \subseteq D$, and all $x \in U$. This condition is satisfied, e.g., by the Green function $G_{D}(\cdot, y)$ in $D \backslash\{y\}$, and it is weaker than regular harmonicity. In this work however, only the notion of regular harmonicity is used. For further discussion, we refer to [35, 40, 48, 81].

\section{Boundary Harnack INEQUALity}

Recall that Assumptions $\mathrm{A}, \mathrm{B}, \mathrm{C}$ and $\mathrm{D}$ are in force throughout the entire paper. Some results, however, hold in greater generality. For example, the following Lemma 3.1 relies solely on Assumption B and (2.9), and it remains true also when $X_{t}$ is a diffusion process. Also, Lemma 3.2 and Corollary 3.3 require Assumptions B and $\mathrm{C}$ but not $\mathrm{A}$ or $\mathrm{D}$.

Lemma 3.1. If $x_{0} \in \mathfrak{X}$ and $0<r<R<\tilde{R}<\infty$, then for all $D \subseteq B\left(x_{0}, R\right)$ we have

$$
\mathbf{P}_{x}\left(X_{\tau_{D}} \in \bar{A}\left(x_{0}, R, \tilde{R}\right)\right) \leq c_{[3.1]} \mathbf{E}_{x} \tau_{D}, \quad x \in B\left(x_{0}, r\right) \cap D,
$$

where $c_{\text {[3.1) }}=c_{\text {[3.1) }}\left(x_{0}, r, R, \tilde{R}\right)=\inf _{\tilde{r}>\tilde{R}} \varrho\left(\bar{A}\left(x_{0}, R, \tilde{R}\right), A\left(x_{0}, r, \tilde{r}\right)\right)$.

Proof. We fix an auxiliary number $\tilde{r}>\tilde{R}$ and $x \in B\left(x_{0}, r\right)$. Let $f \in \mathcal{D}$ be a bump function from Assumption $\mathrm{B}$ for the compact set $\bar{A}\left(x_{0}, R, \tilde{R}\right)$ and the open set $A\left(x_{0}, r, \tilde{r}\right)$. Thus, $f \in \mathcal{D}(\mathcal{A}), f(x)=0, f(y)=1$ for $y \in \bar{A}\left(x_{0}, R, \tilde{R}\right)$ and $0 \leq f(y) \leq 1$ for all $y \in \mathfrak{X}$. By Dynkin's formula (2.11) we have

$$
\mathbf{P}_{x}\left(X_{\tau_{D}} \in \bar{A}\left(x_{0}, R, \tilde{R}\right)\right) \leq \mathbf{E}_{x}\left(f\left(X_{\tau_{D}}\right)\right)-f(x)=G_{D}(\mathcal{A} f)(x) \leq G_{D} \mathbf{1}(x) \sup _{y \in \mathcal{X}} \mathcal{A} f(y) .
$$

Since $G_{D} \mathbf{1}(x)=\mathbf{E}_{x} \tau_{D}$, the proof is complete.

We write $f \approx c g$ if $c^{-1} g \leq f \leq c g$. We will now clarify the relation between BHI and local supremum estimate.

Lemma 3.2. The following conditions are equivalent:

(a) If $x_{0} \in \mathfrak{X}, 0<r<R<R_{0}, D \subseteq B\left(x_{0}, R\right)$ is open, and $f$ is nonnegative, regular harmonic in $D$ and vanishes in $B\left(x_{0}, R\right) \backslash D$, then

$$
f(x) \leq c_{[\sqrt{3.2})} \int_{\mathfrak{X} \backslash B\left(x_{0}, r\right)} f(y) \nu\left(x_{0}, y\right) m(d y)
$$

for $x \in B\left(x_{0}, r\right) \cap D$, where $c_{[3.2)}=c_{[3.2)}\left(x_{0}, r, R\right)$. 
(b) If $x_{0} \in \mathfrak{X}, 0<r<p<q<R<R_{0}, D \subseteq B\left(x_{0}, R\right)$ is open, and $f$ is nonnegative, regular harmonic in $D$ and vanishes in $B\left(x_{0}, R\right) \backslash D$, then

$$
f(x) \approx c_{\sqrt{3.3}} \mathbf{E}_{x}\left(\tau_{D \cap B\left(x_{0}, p\right)}\right) \int_{\mathfrak{X} \backslash B\left(x_{0}, q\right)} f(y) \nu\left(x_{0}, y\right) m(d y)
$$

for $x \in B\left(x_{0}, r\right) \cap D$, where $c_{[3.3)}=c_{[3.3)}\left(x_{0}, r, p, q, R\right)$.

In fact, if (a) holds, then we may let

$$
c_{\text {[3.3) }}\left(x_{0}, r, p, q, R\right)=c_{\underline{3.1]}}\left(x_{0}, r, p, q\right) c_{\underline{3.2}}\left(x_{0}, q, R\right)+c_{[2.7]}\left(x_{0}, p, q\right) \text {, }
$$

and if $(\mathrm{b})$ holds, then we may let

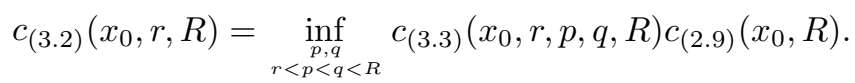

Proof. Since $\mathfrak{X} \backslash B\left(x_{0}, q\right) \subseteq \mathfrak{X} \backslash B\left(x_{0}, r\right)$ and $\mathbf{E}_{x}\left(\tau_{D \cap B\left(x_{0}, p\right)}\right) \leq \mathbf{E}_{x}\left(\tau_{B\left(x_{0}, R\right)}\right) \leq$ $c_{(2.9)}\left(x_{0}, R\right)$, we see that (b) implies (a) with $c_{(3.2)}=c_{(3.3)}\left(x_{0}, r, p, q, R\right) c_{(2.9)}\left(x_{0}, R\right)$. Below we prove the converse. Let (a) hold, and $U=D \cap B\left(x_{0}, p\right)$. We have

$$
f(x)=\mathbf{E}_{x}\left(f\left(X_{\tau_{U}}\right) ; X_{\tau_{U}} \in \bar{B}\left(x_{0}, q\right)\right)+\mathbf{E}_{x}\left(f\left(X_{\tau_{U}}\right) ; X_{\tau_{U}} \in \mathfrak{X} \backslash \bar{B}\left(x_{0}, q\right)\right) .
$$

Denote the terms on the right hand side by $I$ and $J$, respectively. By (3.1) and (3.2),

$$
\begin{aligned}
0 \leq I & \leq \mathbf{P}_{x}\left(X_{\tau_{U}} \in \bar{A}\left(x_{0}, p, q\right)\right) \sup _{y \in B\left(x_{0}, q\right)} f(y) \\
& \leq c_{[3.1} c{ }^{c(3.2)} \mathbf{E}_{x} \tau_{U} \int_{\mathfrak{X} \backslash B\left(x_{0}, q\right)} f(y) \nu\left(x_{0}, y\right) m(d y),
\end{aligned}
$$

with $c_{[3.1)}\left(x_{0}, r, p, q\right)$ and $c_{[3.2)}\left(x_{0}, q, R\right)$. For $J$, the Ikeda-Watanabe formula (2.12) yields

$$
\begin{aligned}
& J=\int_{\mathfrak{X} \backslash \bar{B}\left(x_{0}, q\right)}\left(\int_{U} G_{U}(x, z) \nu(z, y) f(y) m(d z)\right) m(d y)
\end{aligned}
$$

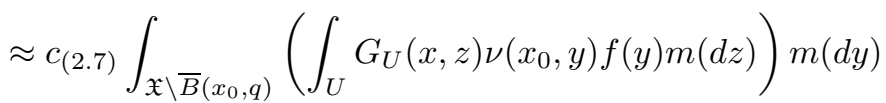

$$
\begin{aligned}
& =c_{\underline{[2.7}} \mathbf{E}_{x} \tau_{U} \int_{\mathfrak{X} \backslash \bar{B}\left(x_{0}, q\right)} \nu\left(x_{0}, y\right) f(y) m(d y),
\end{aligned}
$$

with constant $c_{[2.7}\left(x_{0}, p, q\right)$. Formula (3.3) follows, as we have $c_{[3.1]} c_{[3.2}+c_{[2.7}$ in the upper bound and $1 / c_{(2.7)}$ in the lower bound.

We like to remark that BHI boils down to the approximate factorization (3.3) of $f(x)=\mathbf{P}_{x}\left(X\left(\tau_{D}\right) \in E\right)$. We also note that $\mathbf{P}_{x}\left(X\left(\tau_{D}\right) \in E\right) \approx \nu\left(x_{0}, E\right) \mathbf{E}_{x} \tau_{D}$, if $E$ is far from $B\left(x_{0}, R\right)$, since then $\nu(z, E) \approx \nu\left(x_{0}, E\right)$ in (2.6). However, $\nu(z, E)$ in (2.6) is quite singular and much larger than $\nu\left(x_{0}, E\right)$ if both $z$ and $E$ are close to $\partial B\left(x_{0}, R\right)$. Our main task is to prove that the contribution to (2.6) from such points $z$ is compensated by the relatively small time spent there by $X_{t}^{D}$ when starting at $x \in D$. In fact, we wish to control (2.6) by an integral free from singularities (i.e. (3.2)), if $x$ and $E$ are not too close.

By substituting (3.3) into (1.1), we obtain the following result.

Corollary 3.3. The conditions (a), (b) of Lemma 3.2 imply (BHI) with

$$
c_{\sqrt{1.1)}}\left(x_{0}, r, R\right)=\inf _{\substack{p, q \\ r<p<q<R}}\left(c_{\sqrt{3.3)}}\left(x_{0}, r, p, q, R\right)\right)^{4} \text {. }
$$


The main technical result of the paper is the following local supremum estimate for subharmonic functions, which is of independent interest. The result is proved in Section 4.

Theorem 3.4. Suppose that Assumptions $\mathrm{A}$, B, C] and $\mathrm{D}$ hold true. Let $x_{0} \in \mathfrak{X}$ and $0<r<q<R<R_{0}$, where $R_{0}$ is the localization radius from Assumptions C] and $\mathrm{D}$. Let function $f$ be nonnegative on $\mathfrak{X}$ and regular subharmonic with respect to $X_{t}$ in $B\left(x_{0}, R\right)$. Then

$$
f(x) \leq \int_{\mathfrak{X} \backslash B\left(x_{0}, q\right)} f(y) \pi_{x_{0}, r, q, R}(y) m(d y), \quad x \in B\left(x_{0}, r\right),
$$

where

$$
\pi_{x_{0}, r, q, R}(y)= \begin{cases}c_{(3.9)} \varrho & \text { for } y \in B\left(x_{0}, R\right) \backslash B\left(x_{0}, q\right), \\ 2 c^{(3.9)} \min \left(\varrho, \hat{\nu}\left(y, B\left(x_{0}, R\right)\right)\right) & \text { for } y \in \mathfrak{X} \backslash B\left(x_{0}, R\right),\end{cases}
$$

$\varrho=\varrho\left(\bar{B}\left(x_{0}, q\right), B\left(x_{0}, R\right)\right)$ (see Assumption $\left.\mathbf{B}\right)$, and

$$
c_{\sqrt{(3.9)}}\left(x_{0}, r, q, R\right)=\inf _{p \in(r, q)}\left(c_{\sqrt{2.10)}}\left(x_{0}, r, p, R\right)+\frac{c_{(2.9)}\left(x_{0}, R\right)\left(c_{[2.7}\left(x_{0}, p, q\right)\right)^{2}}{m\left(B\left(x_{0}, p\right)\right)}\right) .
$$

Theorem 3.4 (to be proved in the next section) and Corollary 3.3 lead to BHI. We note that no regularity of the open set $D$ is assumed.

Theorem 3.5. If assumptions $\mathrm{A}, \mathrm{B}, \mathrm{C}$ and $\mathrm{D}$ are satisfied, then $(\mathrm{BHI})$ holds true with

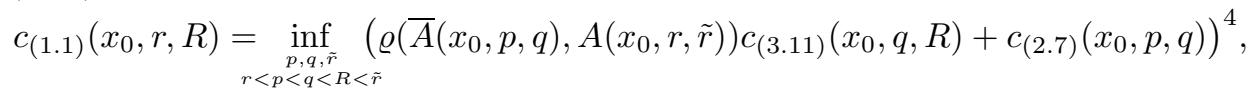

$$
\begin{aligned}
c_{\underline{3.117}}\left(x_{0}, q, R\right)= & \inf _{\substack{\tilde{q} \tilde{R} \\
q<\tilde{q}<R<\tilde{R}}} 2 c_{\underline{\underline{3.9}}}\left(x_{0}, q, \tilde{q}, R\right) \\
& \times \max \left(\frac{\varrho\left(\bar{B}\left(x_{0}, \tilde{q}\right), B\left(x_{0}, R\right)\right)}{c_{\underline{2.8}}\left(x_{0}, \tilde{q}, \tilde{R}\right)}, c_{\underline{2.7}}\left(x_{0}, R, \tilde{R}\right) m\left(B\left(x_{0}, R\right)\right)\right) .
\end{aligned}
$$

Proof. We only need to prove condition (a) of Lemma 3.2 with $c_{(3.2)}$ equal to $c_{\text {(3.11) }}=c_{\text {(3.11) }}\left(x_{0}, r, R\right)$ given above. By (3.7) and (3.8) of Theorem 3.4, it suffices to prove that $\inf _{q \in(r, R)} \sup _{y \in \mathfrak{X} \backslash B\left(x_{0}, q\right)} \pi_{x_{0}, r, q, R}(y) / \nu\left(x_{0}, y\right) \leq c_{\text {(3.11) }}$. For $y \in$ $\bar{A}\left(x_{0}, q, \tilde{R}\right)$ we have

$$
\pi_{x_{0}, r, q, R}(y) \leq 2 c_{\underline{(3.9)}} \varrho \leq \frac{2 c_{(\underline{3.9)}} \varrho}{c_{(2.8)}} \nu\left(x_{0}, y\right),
$$

with $c_{(3.9)}=c_{(3.9)}\left(x_{0}, r, q, R\right), \varrho=\varrho\left(\bar{B}\left(x_{0}, q\right), B\left(x_{0}, R\right)\right)$ and $c_{(2.8)}=c_{(2.8)}\left(x_{0}, q, \tilde{R}\right)$. If $y \in \mathfrak{X} \backslash \bar{B}\left(x_{0}, \tilde{R}\right)$, then

$$
\pi_{x_{0}, r, q, R}(y) \leq 2 c_{\underline{(3.9)}} \hat{\nu}\left(y, B\left(x_{0}, R\right)\right) \leq 2 c_{(\underline{3.9})} c_{(2.7)} m\left(B\left(x_{0}, R\right)\right) \nu\left(x_{0}, y\right),
$$

with $c_{[3.9)}$ as above and $c_{[2.7]}=c_{[2.7)}\left(x_{0}, R, \tilde{R}\right)$. The proof is complete. 
Remark 3.6. (BHI) is said to be scale-invariant if $c_{(1.1)}$ may be so chosen to depend on $r$ and $R$ only through the ratio $r / R$. In some applications, the property plays a crucial role; see, e.g., [14,26]. If $X_{t}$ admits stable-like scaling, then $c_{1.1}$ given by (3.10) is scale-invariant indeed, as explained in Section 5 (see Theorem 5.4).

Remark 3.7. The constant $c_{\overline{1.1}}$ in Theorem 3.5 depends only on basic characteristics of $X_{t}$. Accordingly, in Section 5 it is shown that BHI is stable under small perturbations.

Remark 3.8. BHI applies in particular to hitting probabilities: if $0<r<R<R_{0}$, $x, y \in B\left(x_{0}, r\right) \cap D$ and $E_{1}, E_{2} \subseteq \mathfrak{X} \backslash B\left(x_{0}, R\right)$, then

$$
\mathbf{P}_{x}\left(X_{\tau_{D}} \in E_{1}\right) \mathbf{P}_{y}\left(X_{\tau_{D}} \in E_{2}\right) \leq c_{\underline{1.1}} \mathbf{P}_{y}\left(X_{\tau_{D}} \in E_{1}\right) \mathbf{P}_{x}\left(X_{\tau_{D}} \in E_{2}\right) .
$$

Remark 3.9. BHI implies the usual Harnack inequality if, e.g., constants are harmonic.

The approach to BHI via approximate factorization was applied to isotropic stable processes in [26], to stable-like subordinate diffusion on the Sierpiński gasket in [55], and to a wide class of isotropic Lévy processes in [65]. In all these papers, the taming of the intensity of jumps near the boundary was a crucial step. This parallels the connection of the Carleson estimate and BHI in the classical potential theory; see Section 1

\section{Regularization of the EXit Distribution}

In this section we prove Theorem 3.4. The proof is rather technical, so we begin with a few words of introduction and an intuitive description of the idea of the proof.

In [26, Lemma 6], an analogue of Theorem 3.4 was obtained for the isotropic $\alpha$-stable Lévy processes by averaging the harmonic measure of the ball against the variable radius of the ball. The procedure yields a kernel with no singularities and a mean value property for harmonic functions. In the setting of 26 the boundedness of the kernel follows from the explicit formula and bounds for the harmonic measure of a ball. A similar argument is classical for harmonic functions of the Laplacian and the Brownian motion. For more general processes $X_{t}$ this approach is problematic: while the Ikeda-Watanabe formula gives precise bounds for the harmonic measure far from the ball, satisfactory estimates near the boundary of the ball require exact decay rate of the Green function, which is generally unavailable. In fact, resolved cases indicate that sharp estimates of the Green function are equivalent to BHI (20]), hence not easier to obtain. Below we use a different method to mollify the harmonic measure.

Recall that the harmonic measure of $B$ is the distribution of $X\left(\tau_{B}\right)$. It may be interpreted as the mass lost by a particle moving along the trajectory of $X_{t}$, when it is killed at the moment $\tau_{B}$. In the present paper we let the particle lose the mass gradually before time $\tau_{B}$, with intensity $\psi\left(X_{t}\right)$ for a suitable function $\psi \geq 0$ sharply increasing at $\partial B$. The resulting distribution of the lost mass defines a kernel with a mean value property for harmonic functions, and it is less singular than the distribution of $X\left(\tau_{B}\right)$.

Throughout this section, we fix $x_{0} \in \mathfrak{X}$ and four numbers $0<r<p<q<R<$ $R_{0}$, where $R_{0}$ is defined in Assumptions $\mathrm{C}$ and $\mathrm{D}$. For the compact set $\bar{B}\left(x_{0}, q\right)$ and 


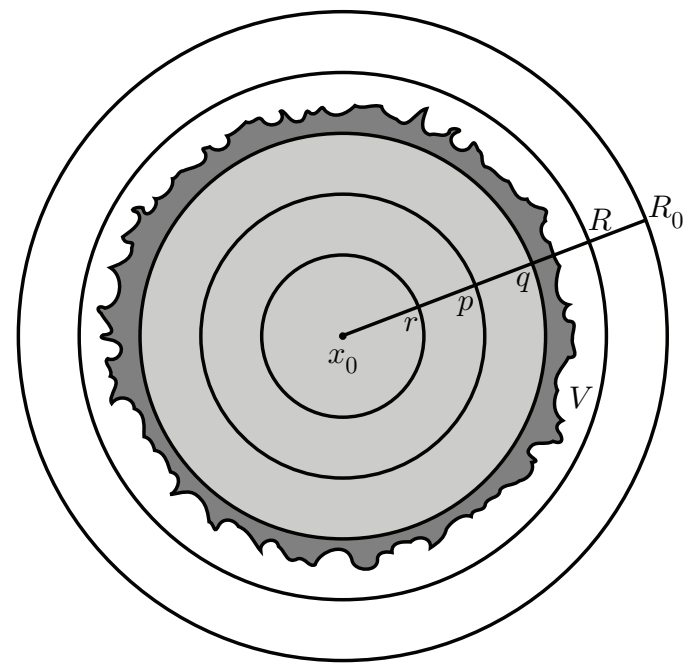

Figure 1. Notation for Section 4 .

the open set $B\left(x_{0}, R\right)$ we consider the bump function $\varphi$ provided by Assumption B. We let

$$
\delta=\sup _{x \in \mathfrak{X}} \max (\mathcal{A} \varphi(x), \hat{\mathcal{A}} \varphi(x))
$$

and

$$
V=\{x \in \mathfrak{X}: \varphi(x)>0\} .
$$

We have $V \subseteq B\left(x_{0}, R\right)$; see Figure 1, By Assumption B $m(\partial V)=0$. Note that $\mathcal{A} \varphi(x) \leq 0$ and $\hat{\mathcal{A}} \varphi(x) \leq 0$ if $x \in B\left(x_{0}, q\right)$, and $\delta$ can be arbitrarily close to $\varrho\left(\bar{B}\left(x_{0}, q\right), B\left(x_{0}, R\right)\right)$.

We consider a function $\psi: \mathfrak{X} \cup\{\partial\} \rightarrow[0, \infty]$ continuous in the extended sense and such that $\psi(x)=\infty$ for $x \in(\mathfrak{X} \backslash V) \cup\{\partial\}$, and $\psi(x)<\infty$ when $x \in V$. Let

$$
A_{t}=\lim _{\varepsilon \searrow 0} \int_{0}^{t+\varepsilon} \psi\left(X_{s}\right) d s, \quad t \geq 0 .
$$

We see that $A_{t}$ is a right-continuous, strong Markov, nonnegative (possibly infinite) additive functional, and $A_{t}=\infty$ for $t \geq \zeta$. We define the right-continuous multiplicative functional

$$
M_{t}=e^{-A_{t}} .
$$

For $a \in[0, \infty]$, we let $\tau_{a}$ be the first time when $A_{t} \geq a$. In particular, $\tau_{\infty}$ is the time when $A_{t}$ becomes infinite. Note that $A_{t}$ and $M_{t}$ are continuous except perhaps at the single (random) moment $\tau_{\infty}$ when $A_{t}$ becomes infinite and the left limit $A\left(\tau_{\infty}-\right)$ is finite. Since $A_{t}$ is finite for $t<\tau_{V}$, we have $\tau_{\infty} \geq \tau_{V}$. If $\psi$ grows sufficiently fast near $\partial V$, then in fact $\tau_{\infty}=\tau_{V}$, as we shall see momentarily.

Lemma 4.1. If $c_{1}, c_{2}>0$ are such that $\psi(x) \geq c_{1}(\varphi(x))^{-1}-c_{2}$ for all $x \in V$, then $A\left(\tau_{V}\right)=\infty$ and $M\left(\tau_{V}\right)=0 \mathbf{P}_{x}$-a.s. for every $x \in \mathfrak{X}$. In particular, $\tau_{V}=\tau_{\infty}$.

Proof. We first assume that $x \in \mathfrak{X} \backslash V$. In this case it suffices to prove that $A_{0}=\infty$. Since $\mathcal{A} \varphi(y) \leq \delta$ for all $y \in \mathfrak{X}$, and $\varphi(x)=0$, from Dynkin's formula 
for the (deterministic) time $s$ it follows that $\mathbf{E}_{x}\left(\varphi\left(X_{s}\right)\right) \leq \delta s$ for all $s>0$. By the Schwarz inequality,

$$
\left(\int_{\varepsilon}^{t} \frac{1}{s} d s\right)^{2} \leq\left(\int_{\varepsilon}^{t} \frac{\varphi\left(X_{s}\right)}{s^{2}} d s\right)\left(\int_{\varepsilon}^{t} \frac{1}{\varphi\left(X_{s}\right)} d s\right)
$$

where $0<\varepsilon<t$. Here we use the conventions $1 / 0=\infty$ and $0 \cdot \infty=\infty$. Thus,

$$
\begin{aligned}
\mathbf{E}_{x}\left(\left(\int_{\varepsilon}^{t} \frac{1}{\varphi\left(X_{s}\right)} d s\right)^{-1}\right) & \leq\left(\int_{\varepsilon}^{t} \frac{1}{s} d s\right)^{-2} \mathbf{E}_{x}\left(\int_{\varepsilon}^{t} \frac{\varphi\left(X_{s}\right)}{s^{2}} d s\right) \\
& \leq\left(\int_{\varepsilon}^{t} \frac{1}{s} d s\right)^{-2} \int_{\varepsilon}^{t} \frac{\delta}{s} d s=\frac{\delta}{\log (t / \varepsilon)},
\end{aligned}
$$

with the convention $1 / \infty=0$. Hence,

$$
\begin{aligned}
\mathbf{E}_{x}\left(\frac{1}{A_{t}+c_{2} t}\right) & \leq \mathbf{E}_{x}\left(\left(\int_{\varepsilon}^{t}\left(\psi\left(X_{s}\right)+c_{2}\right) d s\right)^{-1}\right) \\
& \leq \mathbf{E}_{x}\left(\left(\int_{\varepsilon}^{t} \frac{c_{1}}{\varphi\left(X_{s}\right)} d s\right)^{-1}\right) \leq \frac{\delta}{c_{1} \log (t / \varepsilon)} .
\end{aligned}
$$

By taking $\varepsilon \searrow 0$, we obtain

$$
\mathbf{E}_{x}\left(\frac{1}{A_{t}+c_{2} t}\right)=0 .
$$

It follows that $A_{t}=\infty \mathbf{P}_{x^{-}}$-a.s. We conclude that $A_{0}=\infty$ and $M_{0}=0 \mathbf{P}_{x}$-a.s., as desired.

When $x \in V$, the result in the statement of the lemma follows from the strong Markov property. Indeed, by the definition (4.3) of $A_{t}, A\left(\tau_{V}\right)=A\left(\tau_{V}-\right)+$ $\left(A_{0} \circ \vartheta_{\tau_{V}}\right)$, where $\vartheta_{\tau_{V}}$ is the shift operator on the underlying probability space, which shifts sample paths of $X_{t}$ by the random time $\tau_{V}$, and $A\left(\tau_{V}-\right)$ denotes the left limit of $A_{t}$ at $t=\tau_{V}$. Hence, $M\left(\tau_{V}\right)=M\left(\tau_{V}-\right) \cdot\left(M_{0} \circ \vartheta_{\tau_{V}}\right)$. Furthermore, $X\left(\tau_{V}\right) \in \mathfrak{X} \backslash V \mathbf{P}_{x^{-}}$a.s., so by the first part of the proof, we have $\mathbf{E}_{X\left(\tau_{V}\right)}\left(M_{0}\right)=0$ $\mathbf{P}_{x}$-a.s. Thus,

$$
\mathbf{E}_{x} M_{\tau_{V}}=\mathbf{E}_{x}\left(M_{\tau_{V}}-\mathbf{E}_{X\left(\tau_{V}\right)}\left(M_{0}\right)\right)=0,
$$

which implies that $M\left(\tau_{V}\right)=0 \mathbf{P}_{x^{-}}$a.s. and $A\left(\tau_{V}\right)=\infty \mathbf{P}_{x^{-}}$a.s..

From now on we only consider the case when the assumptions of Lemma 4.1 are satisfied, and $c_{1}, c_{2}$ are reserved for the constants in the condition $\psi(x) \geq$ $c_{1}(\varphi(x))^{-1}-c_{2}$. By the definition and right-continuity of paths of $X_{t}, A_{t}$ and $M_{t}$ are monotone right-differentiable continuous functions of $t$ on $\left[0, \tau_{V}\right)$, with derivatives $\psi\left(X_{t}\right)$ and $-\psi\left(X_{t}\right) M_{t}$, respectively.

Let $\varepsilon_{a}(\cdot)$ be the Dirac measure at $a$. Lemma 4.1 yields the following result.

Corollary 4.2. We have $-d M_{t}=\psi\left(X_{t}\right) M_{t} d t+M\left(\tau_{V}-\right) \varepsilon_{\tau_{V}}(d t) \mathbf{P}_{x^{-}}$a.s. In particular,

$$
-\mathbf{E}_{x} \int_{[0, \tau)} f\left(X_{t}\right) d M_{t}=\mathbf{E}_{x}\left(\int_{0}^{\tau} f\left(X_{t}\right) \psi\left(X_{t}\right) M_{t} d t\right)+\mathbf{E}_{x}\left(M_{\tau_{V}-} f\left(X_{\tau_{V}}\right) ; \tau>\tau_{V}\right)
$$

for any measurable random time $\tau$ and nonnegative or bounded function $f$. 
We emphasize that if $M_{t}$ has a jump at $\tau$, in which case we must have $\tau=\tau_{V}$, then the jump does not contribute to the Lebesgue-Stieltjes integral $\int_{[0, \tau)} f\left(X_{t}\right) d M_{t}$ in (4.5). The same remark applies to (4.6) below.

Recall that $\tau_{a}=\inf \left\{t \geq 0: A_{t} \geq a\right\}$. Note that $\tau_{a}$ are Markov times for $X_{t}$, $a \mapsto \tau_{a}$ is the left-continuous inverse of $t \mapsto A_{t}$, and the events $\left\{t<\tau_{a}\right\}$ and $\left\{A_{t}<a\right\}$ are equal. We have $A\left(\tau_{a}\right)=a$ unless $\tau_{a}=\tau_{V}$, and, clearly, $\tau_{a} \leq \tau_{\infty}=\tau_{V}$.

The following may be considered as an extension of Dynkin's formula.

Lemma 4.3. For $f \in \mathcal{D}(\mathcal{A})$, Markov time $\tau$, and $x \in V$, we have

$$
\mathbf{E}_{x} \int_{0}^{\tau} \mathcal{A} f\left(X_{t}\right) M_{t} d t=\mathbf{E}_{x}\left(f\left(X_{\tau}\right) M_{\tau-}\right)-f(x)-\mathbf{E}_{x} \int_{[0, \tau)} f\left(X_{t}\right) d M_{t} .
$$

If $g=(\mathcal{A}-\psi) f$ and $\tau \leq \tau_{V}$, then

$$
\mathbf{E}_{x} \int_{0}^{\tau} g\left(X_{t}\right) M_{t} d t=\mathbf{E}_{x}\left(f\left(X_{\tau}\right) M_{\tau-}\right)-f(x) .
$$

In fact, (4.6) holds for every strong Markov right-continuous multiplicative functional $M_{t}$.

Proof. Since $\int_{A_{t}}^{\infty} e^{-a} d a=M_{t}$ and $\left\{t<\tau_{a}\right\}=\left\{A_{t}<a\right\}$, by Fubini,

$$
\begin{aligned}
\mathbf{E}_{x} \int_{0}^{\tau} \mathcal{A} f\left(X_{t}\right) M_{t} d t & =\mathbf{E}_{x} \int_{0}^{\tau} \mathcal{A} f\left(X_{t}\right)\left(\int_{0}^{\infty} \mathbf{1}_{\left(0, \tau_{a}\right)}(t) e^{-a} d a\right) d t \\
& =\int_{0}^{\infty}\left(\mathbf{E}_{x} \int_{0}^{\min \left(\tau, \tau_{a}\right)} \mathcal{A} f\left(X_{t}\right) d t\right) e^{-a} d a .
\end{aligned}
$$

Since $\min \left(\tau, \tau_{a}\right)$ is a Markov time for $X_{t}$, we can apply Dynkin's formula. It follows that

$$
\mathbf{E}_{x} \int_{0}^{\min \left(\tau, \tau_{a}\right)} \mathcal{A} f\left(X_{t}\right) d t=\mathbf{E}_{x}\left(f\left(X_{\min \left(\tau, \tau_{a}\right)}\right)\right)-f(x) .
$$

By Fubini and the substitution $\tau_{a}=t, a=A_{t}, e^{-a}=M_{t}$,

$$
\begin{aligned}
\mathbf{E}_{x} \int_{0}^{\tau} \mathcal{A} f\left(X_{t}\right) M_{t} d t & =\int_{0}^{\infty}\left(\mathbf{E}_{x}\left(f\left(X_{\min \left(\tau, \tau_{a}\right)}\right)\right)-f(x)\right) e^{-a} d a \\
& =\mathbf{E}_{x}\left(\int_{0}^{\infty} f\left(X_{\min \left(\tau, \tau_{a}\right)}\right) e^{-a} d a\right)-f(x) \\
& =-\mathbf{E}_{x}\left(\int_{[0, \infty)} f\left(X_{\min (\tau, t)}\right) d M_{t}\right)-f(x) .
\end{aligned}
$$

We emphasize that the last equality holds true also if $\tau=\tau_{V}$ with positive probability. We see that (4.6) holds. By (4.5) we obtain (4.7).

The functional $M_{t}$ is a Feynman-Kac functional, interpreted as the diminishing mass of a particle started at $x \in \mathfrak{X}$. We shall estimate the kernel $\pi_{\psi}(x, d y)$, defined as the expected amount of mass left by the particle at $d y$. Namely, for any nonnegative or bounded $f$ we define

$$
\pi_{\psi} f(x)=-\mathbf{E}_{x} \int_{[0, \infty)} f\left(X_{t}\right) d M_{t}, \quad x \in \mathfrak{X} .
$$


Note that $\pi_{\psi} f(x)=f(x)$ for $x \in \mathfrak{X} \backslash V$. By the substitution $\tau_{a}=t, a=A_{t}$, $e^{-a}=M_{t}$ and Fubini, we obtain that

$$
\pi_{\psi} f(x)=\mathbf{E}_{x}\left(\int_{0}^{\infty} f\left(X_{\tau_{a}}\right) e^{-a} d a\right)=\int_{0}^{\infty} \mathbf{E}_{x}\left(f\left(X_{\tau_{a}}\right)\right) e^{-a} d a .
$$

The potential kernel $G_{\psi}(x, d y)$ of the functional $M_{t}$ will play an important role. Namely, for any nonnegative or bounded $f$ we let

$$
G_{\psi} f(x)=\mathbf{E}_{x} \int_{0}^{\infty} f\left(X_{t}\right) M_{t} d t=\mathbf{E}_{x} \int_{0}^{\infty}\left(\int_{0}^{\tau_{a}} f\left(X_{t}\right) d t\right) e^{-a} d a .
$$

In the second equality above, the identities $M_{t}=\int_{A_{t}}^{\infty} e^{-a} d a$ and $\left\{t<\tau_{a}\right\}=$ $\left\{A_{t}<a\right\}$ were used together with Fubini, as in the proof of Lemma 4.3. We note that $G_{\psi}(x, d y)$ measures the expected time spent by the process $X_{t}$ at $d y$, weighted by the decreasing mass of $X_{t}$ (compare with the similar role of $G_{V}(x, y) m(d y)$ ). There is a semigroup of operators $T_{t}^{\psi} f(x)=\mathbf{E}_{x}\left(f\left(X_{t}\right) M_{t}\right)$ associated with the multiplicative functional $M_{t}$. Furthermore, $T_{t}^{\psi}$ are transition operators of a Markov process $X_{t}^{\psi}$, the subprocess of $X_{t}$ corresponding to $M_{t}$. With the definitions of [18], $M_{t}$ is a strong Markov right-continuous multiplicative functional and $V$ is the set of permanent points for $M_{t}$. Therefore, $X_{t}^{\psi}$ is a standard Markov process with state space $V$; see [18, III.3.12, III.3.13 and the discussion after III.3.17]. (From (4.4) and [18, Proposition III.5.9] it follows that $M_{t}$ is an exact multiplicative functional. Furthermore, since $M_{t}$ can be discontinuous only at $t=\tau_{V}$, the functional $M_{t}$ is quasi-left-continuous in the sense of [18, III.3.14], and therefore $X_{t}^{\psi}$ is a Hunt process on $V$. However, we do not use these properties in our development.)

Informally, $X_{t}^{\psi}$ is obtained from $X_{t}$ by terminating the paths of $X_{t}$ with rate $\psi\left(X_{t}\right) d t$, and $\pi_{\psi}(x, d y)$ is the distribution of $X_{t}$ stopped at the time when $X_{t}^{\psi}$ is killed. Furthermore, $G_{\psi}(x, d y)$ is the potential kernel of $X_{t}^{\psi}$. To avoid technical difficulties related to subprocesses and the domains of their generators, in what follows we rely mostly on the formalism of additive and multiplicative functionals.

The multiplicative functional $\hat{M}_{t}$ is defined just as $M_{t}$, but for the dual process $\hat{X}_{t}$. We correspondingly define $\hat{\pi}_{\psi}$ and $\hat{G}_{\psi}$. Since the paths of $\hat{X}_{t}$ can be obtained from those of $X_{t}$ by time-reversal and $M_{t}$ and $\hat{M}_{t}$ are defined by integrals invariant upon time-reversal, the definition of $\hat{M}_{t}$ agrees with that of [37, formula (13.24)]. Hence, by [37, Theorem 13.25], $M_{t}$ and $\hat{M}_{t}$ are dual multiplicative functionals. It follows that the subprocess $\hat{X}_{t}^{\psi}$ of $\hat{X}_{t}$ corresponding to the multiplicative functional $\hat{M}_{t}$ is the dual process of $X_{t}^{\psi}$; see [37, 13.6 and Remark 13.26]. Hence, the potential kernel $G_{\psi}$ of $X_{t}^{\psi}$ admits a uniquely determined density function $G_{\psi}(x, y)(x, y \in V)$, which is excessive in $x$ with respect to the transition semigroup $T_{t}^{\psi}$ of $X_{t}^{\psi}$, and excessive in $y$ with respect to the transition semigroup $\hat{T}_{t}^{\psi}$ of $\hat{X}_{t}^{\psi}$. Furthermore, $\hat{G}_{\psi}(x, y)=G_{\psi}(y, x)$ is the density of the potential kernel of $\hat{X}_{t}^{\psi}$. Since $G_{\psi}(x, d y)$ is concentrated on $V$, we let $G_{\psi}(x, y)=0$ if $x \in \mathfrak{X} \backslash V$ or $y \in \mathfrak{X} \backslash V$. Clearly, $G_{\psi}(x, d y)$ is dominated by $G_{V}(x, d y)$ for all $x \in V$, and therefore

$$
G_{\psi}(x, y) \leq G_{V}(x, y), \quad x, y \in \mathfrak{X} .
$$

There are important relations between $\pi_{\psi}, G_{\psi}, \psi$ and $\mathcal{A}$. If $f$ is nonnegative or bounded and vanishes in $\mathfrak{X} \backslash V$, then by Corollary 4.2 we have

$$
\pi_{\psi} f(x)=G_{\psi}(\psi f)(x), \quad x \in V .
$$


Considering $\tau=\tau_{V}$, we note that $M\left(\tau_{V}\right)=0$, and so for bounded or nonnegative $f$,

$$
\int_{\left[0, \tau_{V}\right]} f\left(X_{t}\right) d M_{t}=\int_{\left[0, \tau_{V}\right)} f\left(X_{t}\right) d M_{t}-f\left(X_{\tau_{V}}\right) M_{\tau_{V}-} .
$$

If $f \in \mathcal{D}(\mathcal{A})$, then formula (4.6) gives

$$
G_{\psi} \mathcal{A} f(x)=\pi_{\psi} f(x)-f(x), \quad x \in V .
$$

Furthermore, by (4.7), for $f \in \mathcal{D}(\mathcal{A})$ we have

$$
G_{\psi}(\mathcal{A}-\psi) f(x)=\mathbf{E}_{x}\left(f\left(X_{\tau_{V}}\right) M_{\tau_{V}-}\right)-f(x), \quad x \in V .
$$

In particular, if $f \in \mathcal{D}(\mathcal{A})$ vanishes outside of $V$, then we have

$$
G_{\psi}(\mathcal{A}-\psi) f(x)=-f(x), \quad x \in V
$$

(which also follows directly from (4.11) and (4.12)). Formula (4.13) means that the generator of $X_{t}^{\psi}$ agrees with $\mathcal{A}-\psi$ on the intersection of the respective domains.

We now introduce the Green operators $G_{U}^{\psi}$ and harmonic measures $\pi_{U}^{\psi}$ for $X_{t}^{\psi}$. Let $U$ be an open subset of $V$. For nonnegative or bounded $f$ and $x \in V$, we let

$$
\pi_{U}^{\psi} f(x)=\mathbf{E}_{x}\left(f\left(X_{\tau_{U}}\right) M_{\tau_{U}-}\right), \quad G_{U}^{\psi} f(x)=\mathbf{E}_{x} \int_{0}^{\tau_{U}} f\left(X_{t}\right) M_{t} d t .
$$

We note that $G_{V}^{\psi} f=G_{\psi} f$. Also, $\pi_{V}^{\psi} f=\pi_{\psi} f$, if $f$ vanishes in $V$. Furthermore, $G_{U}^{\psi}$ admits a density function $G_{U}^{\psi}(x, y)$, and we have $G_{U}^{\psi}(x, y) \leq G_{U}(x, y), G_{U}^{\psi}(x, y) \leq$ $G_{\psi}(x, y)$. If $f$ vanishes outside of $V$, then we can replace $M\left(\tau_{U}-\right)$ by $M\left(\tau_{U}\right)$ in the definition of $\pi_{U}^{\psi}$. By (4.7), for any $f \in \mathcal{D}(\mathcal{A})$ we have

$$
\pi_{U}^{\psi} f(x)=G_{U}^{\psi}(\mathcal{A}-\psi) f(x)+f(x), \quad x \in V .
$$

In particular, by an approximation argument,

$$
\pi_{U}^{\psi}(x, E)=\int_{U} G_{U}^{\psi}(x, y) \nu(y, E) m(d y), \quad x \in U, E \subseteq \mathfrak{X} \backslash \bar{U} .
$$

Formulas (4.14) and (4.15) can be viewed correspondingly as Dynkin's formula applied to the first exit time, and the Ikeda-Watanabe formula for $X_{t}^{\psi}$.

Recall that $x_{0} \in \mathfrak{X}, 0<r<p<q<R<R_{0}, B\left(x_{0}, q\right) \subseteq V \subseteq B\left(x_{0}, R\right)$ (see Figure 1), $\varphi \in \mathcal{D}$ is positive in $V$ and vanishes in $\mathfrak{X} \backslash V$, and $\varphi(x)=1$ for $x \in B\left(x_{0}, q\right)$.

Lemma 4.4. Let $U=V \backslash \bar{B}\left(x_{0}, q\right)$. If $(\mathcal{A}-\psi) \varphi(x) \leq 0$ for $x \in V$, then

$$
\pi_{U}^{\psi}(x, V \backslash U) \leq \varphi(x), \quad x \in U .
$$

Proof. By (4.14), for $x \in U$ we have

$$
\pi_{U}^{\psi} \varphi(x)-\varphi(x)=G_{U}^{\psi}(\mathcal{A}-\psi) \varphi(x) \leq 0 .
$$

It remains to note that $\varphi=1$ on $V \backslash U$.

Essentially, we use here (and later on) superharmonicity of $\varphi$ with respect to $\mathcal{A}-\psi$. 
Lemma 4.5. If $(\mathcal{A}-\psi) \varphi(x) \leq 0$ for $x \in V$, then

$$
G_{\psi}(x, y) \leq c_{4.17\rceil} \varphi(x), \quad x \in V \backslash B\left(x_{0}, p\right), y \in B\left(x_{0}, r\right),
$$

where

$$
c_{\sqrt{(4.17)}}=c_{\sqrt{4.17}}\left(x_{0}, r, p, q, R\right)=c_{\sqrt{2.10)}}\left(x_{0}, r, p, R\right)+\frac{c_{(2.9)}\left(x_{0}, R\right)\left(c_{(2.7)}\left(x_{0}, p, q\right)\right)^{2}}{m\left(B\left(x_{0}, p\right)\right)} .
$$

Proof. Let $U=V \backslash \bar{B}\left(x_{0}, q\right)$ and $x \in U$. Let $f$ be a nonnegative function supported in $B\left(x_{0}, r\right), \int f(y) m(d y)=1$ and $g(z)=G_{\psi} f(z)$ (this is done to regularize $\left.G_{\psi}(x, y)\right)$. Using the definition of $G_{\psi}$, the relation $f\left(X_{t}\right)=0$ for $t<\tau_{U}$ and the strong Markov property, we obtain that

$$
g(x)=\mathbf{E}_{x}\left(\int_{\tau_{U}}^{\infty} f\left(X_{t}\right) M_{t} d t\right)=\mathbf{E}_{x}\left(g\left(X_{\tau_{U}}\right) M_{\tau_{U}}\right)=\pi_{U}^{\psi} g(x) .
$$

We split the last expectation into two parts, corresponding to the events $X\left(\tau_{U}\right) \in$ $B\left(x_{0}, p\right)$ and $X\left(\tau_{U}\right) \in \bar{A}\left(x_{0}, p, q\right)$ respectively. By (2.10) and the inequality $M\left(\tau_{U}\right)$ $\leq 1$, we have $g(z) \leq c_{(2.10)}\left(x_{0}, r, p, R\right)$ for $z \in \bar{A}\left(x_{0}, p, q\right)$. From (4.16) it follows that

$$
\pi_{U}^{\psi}\left(g \mathbf{1}_{\bar{A}\left(x_{0}, p, q\right)}\right)(x) \leq c_{(\underline{2.10})} \pi_{U}^{\psi}\left(x, \bar{B}\left(x_{0}, q\right)\right) \leq c_{(\underline{2.10})} \varphi(x) .
$$

For the other part, we use (4.15) and (2.7):

$$
\begin{aligned}
\pi_{U}^{\psi}\left(g \mathbf{1}_{B\left(x_{0}, p\right)}\right)(x) & =\int_{U}\left(\int_{B\left(x_{0}, p\right)} g(z) \nu(y, z) m(d z)\right) G_{U}^{\psi}(x, y) m(d y) \\
& \leq c_{\underline{(2.7)}} \int_{U} \nu\left(y, x_{0}\right) G_{U}^{\psi}(x, y) m(d y) \cdot \int_{B\left(x_{0}, p\right)} g(z) m(d z),
\end{aligned}
$$

with constant $c_{(2.7)}\left(x_{0}, p, q\right)$. Again using (2.7) and (4.15), and then (4.16), we obtain

$$
\begin{aligned}
\int_{U} \nu\left(y, x_{0}\right) G_{U}^{\psi}(x, y) m(d y) & \leq \frac{c_{[2.7}}{m\left(B\left(x_{0}, p\right)\right)} \int_{U} \nu\left(y, B\left(x_{0}, p\right)\right) G_{U}^{\psi}(x, y) m(d y) \\
& =\frac{c_{[2.7}}{m\left(B\left(x_{0}, p\right)\right)} \pi_{U}^{\psi}\left(x, B\left(x_{0}, p\right)\right) \leq \frac{c_{(2.7)} \varphi(x)}{m\left(B\left(x_{0}, p\right)\right)} .
\end{aligned}
$$

By (2.9), we have

$$
\begin{aligned}
\int_{B\left(x_{0}, p\right)} g(z) m(d z) & \leq \int_{B\left(x_{0}, r\right)}\left(\int_{B\left(x_{0}, p\right)} G_{V}(z, y) m(d z)\right) f(y) m(d y) \\
& \leq \int_{B\left(x_{0}, r\right)} \hat{\mathbf{E}}_{y}\left(\hat{\tau}_{V}\right) f(y) m(d y) \leq c_{\underline{(2.9)}}
\end{aligned}
$$

with constant $c_{\underline{2.9}}\left(x_{0}, R\right)$. Hence,

$$
\pi_{U}^{\psi}\left(g \mathbf{1}_{B\left(x_{0}, p\right)}\right)(x) \leq \frac{\left(c_{[2.77}\right)^{2} c_{(2.9)} \varphi(x)}{m\left(B\left(x_{0}, p\right)\right)} .
$$

This and (4.18) yield that $g(x) \leq c_{4.17} \varphi(x)$, with $c_{4.17}$ given in the statement of the lemma. 
Recall that $g=G_{\psi} f$, where $f$ is an arbitrary nonnegative function vanishing outside $B\left(x_{0}, r\right)$ with integral equal to 1 . Hence, by approximation, for each $x \in$ $\mathfrak{X} \backslash \bar{B}\left(x_{0}, q\right)$, formula (4.17) holds for almost every $y \in B\left(x_{0}, r\right)$. By Proposition 2.2 (applied to $\left.X_{t}^{\psi}\right)$, (4.17) holds for every $y \in B\left(x_{0}, r\right)$.

For $x \in \bar{A}\left(x_{0}, p, q\right)$, the result follows easily from (2.10). Indeed, we have $G_{\psi}(x, y) \leq G_{V}(x, y) \leq c_{(2.10)}=c_{(2.10)} \varphi(x)$, with constant $c_{[2.10)}\left(x_{0}, r, p, R\right)$. Hence, formula (4.17) holds also for $x \in \bar{A}\left(x_{0}, p, q\right)$, with the same constant.

The above arguments can be repeated for the dual process $\hat{X}_{t}$. Hence, the dual versions of Lemmas 4.4 and 4.5 hold true, with the same $c_{4.177}$.

We are very close to the estimate of $\pi_{\psi}(x, d y)$ for $x \in B\left(x_{0}, r\right)$. Indeed, for $y \in V$ we have $\pi_{\psi}(x, d y)=G_{\psi}(x, y) \psi(y) m(d y)$ (see (4.11) ). When $y \in \mathfrak{X} \backslash \bar{V}$, then, at least heuristically, $\pi_{\psi}(x, d y)=\hat{\mathcal{A}} G_{\psi}^{x}(y) m(d y)$, where $G_{\psi}^{x}(y)=G_{\psi}(x, y)$ vanishes outside of $V$ (see (4.12) ). This will give satisfactory bounds when $y \in \mathfrak{X} \backslash V$. Before we proceed, we first show that $\pi_{\psi}(x, \partial V)=0$.

Lemma 4.6. Suppose that for some $c_{3}, c_{4}>0$, we have $\psi(x) \geq c_{3}+(\varphi(x))^{-1} \hat{\mathcal{A}} \varphi(x)$ and $\psi(x) \leq c_{4} / \varphi(x)$ for $x \in V$. Then for every nonnegative function $f$ we have

$$
c_{3} \int_{V} \pi_{\psi} f(x) \varphi(x) m(d x) \leq c_{4} \int_{V} f(x) m(d x)+\int_{\mathfrak{X} \backslash V} f(x) \hat{\mathcal{A}} \varphi(x) m(d x) .
$$

Proof. First, suppose that $f \in \mathcal{D}(\mathcal{A})$. Denote $h(x)=-(\hat{\mathcal{A}}-\psi) \varphi(x)$ for $x \in V$. Note that $h$ is nonnegative. Let $g(x)=\pi_{\psi} f(x)$ for $x \in \mathfrak{X}$, and hence $g(x)=f(x)$ for $x \in \mathfrak{X} \backslash V$; see (4.8). By (4.12), we have $g(x)=f(x)+G_{\psi} \mathcal{A} f(x)$ for $x \in V$. Hence,

$$
\int_{V} g(x) h(x) m(d x)=\int_{V} f(x) h(x) m(d x)+\int_{V} G_{\psi} \mathcal{A} f(x) h(x) m(d x) .
$$

For the second term, we have

$$
\int_{V} G_{\psi} \mathcal{A} f(x) h(x) m(d x)=\int_{V} \mathcal{A} f(x) \hat{G}_{\psi} h(x) m(d x) .
$$

By (4.13) (the dual version), $\hat{G}_{\psi} h(x)=-\hat{G}_{\psi}(\hat{\mathcal{A}}-\psi) \varphi(x)=\varphi(x)$ for $x \in V$. Hence,

$$
\int_{V} G_{\psi} \mathcal{A} f(x) h(x) m(d x)=\int_{V} \mathcal{A} f(x) \varphi(x) m(d x)=\int_{\mathfrak{X}} f(x) \hat{\mathcal{A}} \varphi(x) m(d x) .
$$

In the last equality, we used the fact that $\varphi(x)=0$ for $x \in \mathfrak{X} \backslash V$. It follows that

$$
\int_{V} g(x) h(x) m(d x)=\int_{V} f(x) h(x) m(d x)+\int_{\mathfrak{X}} f(x) \hat{\mathcal{A}} \varphi(x) m(d x) .
$$

But $h(x)=-(\hat{\mathcal{A}}-\psi) \varphi(x)$, so that finally, after simplification,

$$
\int_{V} g(x) h(x) m(d x)=\int_{V} f(x) \varphi(x) \psi(x) m(d x)+\int_{\mathfrak{X} \backslash V} f(x) \hat{\mathcal{A}} \varphi(x) m(d x) .
$$

Using the inequalities $\psi(x) \varphi(x) \leq c_{4}$ for $x \in V$ and $h(x)=\psi(x) \varphi(x)-\hat{\mathcal{A}} \varphi(x) \geq$ $c_{3} \varphi(x)$ for $x \in V$, we obtain (4.19). The general case of nonnegative $f$ (not necessarily in $\mathcal{D}(\mathcal{A})$ ) follows by approximation.

Lemma 4.7. Suppose that for some $c_{3}, c_{4}>0$, we have $\psi(x) \geq c_{3}+(\varphi(x))^{-1} \hat{\mathcal{A}} \varphi(x)$ and $\psi(x) \leq c_{4} / \varphi(x)$ for $x \in V$. Then $M\left(\tau_{V}-\right) \mathbf{1}_{\partial V}\left(X\left(\tau_{V}\right)\right)=0 \mathbf{P}_{x}$-a.s. and $\pi_{\psi}(x, \partial V)=0$ for all $x \in V$. 
Proof. For $x \in V$ define $g(x)=\pi_{\psi}(x, \partial V)$. By (4.19), $\int_{V} g(x) \varphi(x) m(d x)=0$, so that $g$ vanishes almost everywhere in $V$. We claim that $g$ is excessive for the transition semigroup $T_{t}^{\psi}$ of $X_{t}^{\psi}$. Indeed, we have

$$
g(x)=\mathbf{E}_{x}\left(M\left(\tau_{V}-\right) ; X\left(\tau_{V}\right) \in \partial V\right),
$$

so that by the Markov property, for any $t>0$ and $x \in V$,

$$
\mathbf{E}_{x}\left(M_{t} g\left(X_{t}\right)\right)=\mathbf{E}_{x}\left(M_{t} g\left(X_{t}\right) ; t<\tau_{V}\right)=\mathbf{E}_{x}\left(M_{\tau_{V}-} ; X_{\tau_{V}} \in \partial V, t<\tau_{V}\right) .
$$

The right hand side does not exceed $g(x)$, and by monotone convergence, it converges to $g(x)$ as $t \searrow 0$. Hence $g$ is an excessive function equal to zero almost everywhere in $V$. By [18, Proposition II.3.2 (or by Proposition 2.2), $g(x)=0$ for all $x \in V$.

Recall that according to the remark following Lemma 4.1 we keep assuming that $\psi(x) \geq c_{1}(\varphi(x))^{-1}-c_{2}$ for $x \in V$. Consider $\tilde{\psi}(x)=c_{1}^{-1} \delta\left(\psi(x)+c_{2}\right)+c_{3}$ for some $c_{3}>0$, and let $\tilde{M}_{t}$ be the multiplicative functional defined in a similar manner as $M_{t}$, but with $\psi$ replaced by $\tilde{\psi}$. Clearly, for all $t>0$ we have $M_{t}=0$ if and only if $\tilde{M}_{t}=0$. Since $\tilde{\psi}(x) \geq c_{3}+\delta / \varphi(x)$, an application of Lemma 4.7 to $\tilde{\psi}$ yields the following result.

Corollary 4.8. Suppose that for some $c>0$, we have $\psi(x) \leq c / \varphi(x)$ for $x \in V$. Then $M\left(\tau_{V}-\right) \mathbf{1}_{\partial V}\left(X\left(\tau_{V}\right)\right)=0 \mathbf{P}_{x}$-a.s. for $x \in V$. In particular, $\pi_{\psi}(x, \partial V)=0$ for $x \in V$.

Now we make the actual choice of $\psi$.

Lemma 4.9. Let $\delta$ be given by (4.1), and

$$
\psi(x)=\frac{\max (\mathcal{A} \varphi(x), \hat{\mathcal{A}} \varphi(x), \delta(1-\varphi(x)))}{\varphi(x)}, \quad x \in \mathfrak{X} \cup\{\partial\},
$$

where $1 / 0=\infty$. For all $x \in B\left(x_{0}, r\right)$ we have $\pi_{\psi}(x, d y) \leq \tilde{\pi}_{\psi}(y) m(d y)$, where

$$
\tilde{\pi}_{\psi}(y)=c_{\text {4.17) }}\left(\delta \mathbf{1}_{V \backslash B\left(x_{0}, q\right)}(y)+2 \min (\delta, \hat{\nu}(y, V)) \mathbf{1}_{\mathfrak{X} \backslash V}(y)\right)
$$

with $c_{\text {4.177 }}=c_{\text {[4.17) }}\left(x_{0}, r, p, q, R\right)$ given in Lemma 4.5.

Proof. Note that $\psi(x) \geq \delta(\varphi(x))^{-1}-\delta,(\mathcal{A}-\psi) \varphi(x) \leq 0,(\hat{\mathcal{A}}-\psi) \varphi(x) \leq 0$ and $\psi(x) \leq \delta / \varphi(x)$ for $x \in V$. Hence, we may apply Lemmas 4.1, 4.4 and 4.5, Corollary 4.8, and their dual versions. By Corollary 4.8, $\pi_{\psi}(x, \partial V)=0$ for all $x \in V$. Since $\mathcal{A} \varphi(x) \leq 0$ and $\hat{\mathcal{A}} \varphi(x) \leq 0$ for $x \in B\left(x_{0}, q\right)$, we have $\psi(x)=0$ for $x \in B\left(x_{0}, q\right)$, and therefore $\pi_{\psi}\left(x, B\left(x_{0}, q\right)\right)=0$ for all $x \in V$.

Fix $x \in B\left(x_{0}, r\right)$. If $f$ is nonnegative and vanishes in $B\left(x_{0}, q\right)$ and in $\mathfrak{X} \backslash V$, then (4.11) yields that

$$
\pi_{\psi} f(x)=G_{\psi}(\psi f)(x)=\int_{V \backslash B\left(x_{0}, q\right)} G_{\psi}(x, y) \psi(y) f(y) m(d y) .
$$

Using (4.17) for $\hat{G}_{\psi}$ and the inequality $\varphi(y) \psi(y) \leq \delta$ for $y \in V$, we have

$$
\pi_{\psi} f(x) \leq c_{\underline{4.17}]} \int_{V \backslash B\left(x_{0}, q\right)} \varphi(y) \psi(y) f(y) m(d y) \leq c_{\underline{4.17]}} \delta \int_{V \backslash B\left(x_{0}, q\right)} f(y) m(d y),
$$


with constant $c_{4.17}\left(x_{0}, r, p, q, R\right)$. Suppose now that $f \in \mathcal{D}(\mathcal{A})$ vanishes in $V$. By (4.12),

$$
\begin{aligned}
\pi_{\psi} f(x) & =G_{\psi} \mathcal{A} f(x)=\int_{V} G_{\psi}(x, y)\left(\int_{\mathfrak{X} \backslash V} f(z) \nu(y, z) m(d z)\right) m(d y) \\
& =\int_{\mathfrak{X} \backslash V}\left(\int_{V} G_{\psi}(x, y) \nu(y, z) m(d y)\right) f(z) m(d z) .
\end{aligned}
$$

We estimate the inner integral for $z \in \mathfrak{X} \backslash V$. Using (4.17) for $\hat{G}_{\psi}$, we have

$$
\begin{aligned}
\int_{V \backslash B\left(x_{0}, p\right)} G_{\psi}(x, y) \nu(y, z) m(d y) & \leq c_{4.17} \int_{V \backslash B\left(x_{0}, p\right)} \varphi(y) \nu(y, z) m(d y) \\
& =c_{4.17} \hat{\mathcal{A}} \varphi(z) .
\end{aligned}
$$

The integral over $B\left(x_{0}, p\right)$ is estimated as in the proof of Lemma 4.5.

$$
\begin{aligned}
\int_{B\left(x_{0}, p\right)} G_{\psi}(x, y) \nu(y, z) m(d y) & \leq c_{[2.7)} \nu\left(x_{0}, z\right) \int_{B\left(x_{0}, p\right)} G_{V}(x, y) m(d y) \\
& \leq c_{[2.7]} \nu\left(x_{0}, z\right) \mathbf{E}_{x} \tau_{V} \leq c_{[2.7]} c_{[2.9]} \nu\left(x_{0}, z\right) \\
& \leq \frac{c_{[2.9)}\left(c_{(2.7)}\right)^{2}}{m\left(B\left(x_{0}, p\right)\right)} \hat{\nu}\left(z, B\left(x_{0}, p\right)\right) \leq c_{[4.17} \hat{\mathcal{A}} \varphi(z),
\end{aligned}
$$

with constants $c_{[2.7]}\left(x_{0}, p, q\right), c_{[2.9)}\left(x_{0}, R\right)$ and $c_{[4.17}\left(x_{0}, r, p, q, R\right)$. Since $\hat{\mathcal{A}} \varphi(z) \leq$ $\delta$ and $\hat{\mathcal{A}} \varphi(z) \leq \hat{\nu}(z, V)$, we obtain that

$$
\pi_{\psi} f(x) \leq 2 c_{\underline{4.17} \mathrm{~J}} \int_{\mathfrak{X} \backslash V} f(z) \hat{\mathcal{A}} \varphi(z) m(d z) \leq 2 c_{\underline{4.17}} \int_{\mathfrak{X} \backslash V} f(z) \min (\delta, \hat{\nu}(z, V)) m(d z) .
$$

By approximation, (4.23) holds for any nonnegative $f$ vanishing in $\bar{V}$. Formula (4.21) is a combination of (4.22), (4.23), $\pi_{\psi}(x, \partial V)=0$ and $\pi_{\psi}\left(x, B\left(x_{0}, q\right)\right)=0$ for all $x \in V$.

Lemma 4.10. If a nonnegative function $f$ is regular subharmonic in $B\left(x_{0}, R\right)$, then $f(x) \leq \pi_{\psi} f(x)$ for $x \in B\left(x_{0}, r\right)$. If $f$ is regular harmonic, then equality holds.

Proof. If $f$ is regular subharmonic in $V$, then $f(x) \leq \mathbf{E}_{x}\left(f\left(X\left(\tau_{a}\right)\right)\right)$ for all $a \in$ $[0, \infty]$. If $f$ is regular harmonic in $V$, then equality holds. The result follows by (4.9).

The local maximum estimate is now proved as follows.

Proof of Theorem 3.4. Fix $p \in(r, q)$. Choose $\varepsilon>0$ and $\varphi$ as in the beginning of this section, and so that $\delta=\sup _{x \in \mathfrak{X}} \max (\mathcal{A} \varphi(x), \hat{\mathcal{A}} \varphi(x))<\varrho\left(\bar{B}\left(x_{0}, q\right), B\left(x_{0}, R\right)\right)+\varepsilon$. Define $\psi$ as in (4.20). By Lemmas 4.9 and 4.10, we have (3.7) with $\pi_{x_{0}, r, q, R}(y)$ bounded from above by $\pi_{\psi}(y)$ defined in (4.21). Note that $\hat{\nu}(y, V) \leq \hat{\nu}\left(y, B\left(x_{0}, R\right)\right)$. Since $\varepsilon>0$ and $p \in(r, q)$ are arbitrary, formulas (3.8) and (3.9) follow.

We conclude this section with a result on diffusion processes. The above argument remains valid when $\nu$ vanishes everywhere, i.e., $X_{t}$ is a diffusion process. In this case (2.9) is not a consequence of Assumption C] so we need to add (2.9) as an assumption. No other changes in the argument are needed, and in fact the proof of Lemma 4.5 simplifies significantly, since $X_{t}$ exits $U$ through the boundary of $U$, 
and therefore $X\left(\tau_{U}\right)$ is never in $B\left(x_{0}, p\right)$. Therefore, we have proved the following result.

Theorem 4.11. Assume that $X_{t}$ is a diffusion process satisfying Assumptions $\mathrm{A}$, B and D, and formula (2.9). Let $x_{0} \in \mathfrak{X}$ and $0<r<q<R<R_{0}$, where $R_{0}$ is the localization radius of (2.9) and Assumption $\mathrm{D}$. Let $f$ be a nonnegative function on $B\left(x_{0}, R\right)$, regular subharmonic in $B\left(x_{0}, R\right)$ with respect to $X_{t}$. Then

$$
f(x) \leq c_{\underline{4.24}} \int_{\bar{A}\left(x_{0}, q, R\right)} f(y) m(d y), \quad x \in B\left(x_{0}, r\right) .
$$

Here $c_{[4.24)}=c_{(4.24)}\left(x_{0}, r, q, R\right)=c_{(2.10)} \delta$, where $\delta=\varrho\left(\bar{B}\left(x_{0}, q\right), B\left(x_{0}, R\right)\right)$ and $c_{\underline{(2.10)}}=c_{\underline{2.10)}}\left(x_{0}, r, q, R\right)$ are defined in Assumptions $\mathrm{B}$ and $\mathrm{D}$.

Remark 4.12. For diffusion processes, the local supremum estimate (4.24) for subharmonic functions is typically proved analytically, using Sobolev embeddings and Moser iteration; see, e.g., 42. Theorem 3.4 requires more regularity of the process $X_{t}$ as compared to the analytical approach because we assume the existence of bump functions in the domain of the Feller generator (Assumption B), while Moser iteration is based on the energy form. However, our approach does not depend on Sobolev embeddings, and so it applies also to Sierpiński carpets and some other highly irregular state spaces $\mathfrak{X}$. It would be interesting to find an analytical proof of the local supremum estimate for jump-type processes, which would not require Assumption B. Related results have been recently studied when the Lévy kernel $\nu(x, y)$ is comparable to $(d(x, y))^{-d-\alpha}$ (see [56] and the references therein). Further comments on this subject are given in Example 5.6 and Appendix A.

\section{EXTENSIONS AND EXAMPLES}

In this section we study several applications of our boundary Harnack inequality, and discuss limitations of Theorem 3.5. We sketch the range of possible applications by indicating rather general classes of processes satisfying the assumptions of Theorem 3.5, without getting into technical details. Before that, however, we discuss an important notion of scale-invariance introduced in Remark 3.6. This property can be proved in a fairly general setting, which we call stable-like scaling.

Definition 5.1. The process $X_{t}$ is said to have stable-like scaling property with dimension $n>0$, index $\alpha>0$ and localization radius $R_{0} \in(0, \infty](\alpha$-stable-like scaling for short), if the following conditions are met:

(a) $\mathfrak{X}$ is locally an Ahlfors regular $n$-space; that is, $c^{-1} r^{n} \leq m(B(x, r)) \leq c r^{n}$ when $0<r<R_{0}$ and $x \in \mathfrak{X}$;

(b) $c_{[2.7]}\left(x_{0}, r, R\right) \leq c(r / R)$ when $0<r<R<R_{0}, x_{0} \in \mathfrak{X}$ in the relative constancy of the Lévy measure condition in Assumption $\mathrm{C}$.

(c) $c_{(2.8)}\left(x_{0}, r, R\right) \geq c(r / R) R^{-n-\alpha}$ when $0<r<R<R_{0}, x_{0} \in \mathfrak{X}$; that is, $\nu(x, y) \geq c(d(x, y))^{-n-\alpha}$ when $d(x, y)<R_{0}$;

(d) $c_{(2.9)}\left(x_{0}, r\right) \leq c r^{\alpha}$ when $0<r<R_{0}, x_{0} \in \mathfrak{X}$ in the upper bound for mean exit time from a ball;

(e) $c_{\underline{2.10}}\left(x_{0}, r, p, R\right) \leq c(r / R, p / R) R^{\alpha-n}$ when $0<r<p<R<R_{0}$ and $x_{0} \in \mathfrak{X}$ in the off-diagonal upper bound for the Green function of a ball;

(f) $\varrho\left(\bar{B}\left(x_{0}, r\right), B\left(x_{0}, R\right)\right) \leq c(r / R) R^{-\alpha}$ when $0<r<R<R_{0}$ and $x_{0} \in \mathfrak{X}$, and $\varrho\left(\bar{A}\left(x_{0}, p, R\right), A\left(x_{0}, r, \tilde{r}\right)\right) \leq c(r / R, p / R, R / \tilde{r}) R^{-\alpha}$ when $0<r<p<R<\tilde{r}$ in Assumption B. 
Proposition 5.2. If the scaling property (a) is satisfied, then conditions (b), (c) and $(\mathrm{d})$ are consequences of:

(g) the Lévy kernel of $X_{t}$ satisfies

$$
c^{-1}(d(x, y))^{-n-\alpha} \exp (-q d(x, y)) \leq \nu(x, y) \leq c(d(x, y))^{-n-\alpha} \exp (-q d(x, y))
$$

for some $q \geq 0$ and for all $x, y \in \mathfrak{X}$.

Note that the same parameter $q$ appears in the lower and the upper bound.

Proof. Conditions (b) and (c) follow directly from (g), Furthermore, by (a) and the triangle inequality, there is $R_{0}>0$ such that if $x_{0} \in \mathfrak{X}$ and $0<r<R_{0}$, then for some $y \in B\left(x_{0}, c_{1} r\right) \backslash B\left(x_{0}, r\right)$ where $c_{1}>2$, the balls $B\left(x_{0}, r\right)$ and $B(y, r)$ are disjoint. Hence, for all $x \in B\left(x_{0}, r\right)$ we have by (a) and (g).

$$
\nu\left(x, \mathfrak{X} \backslash B\left(x_{0}, r\right)\right) \geq \nu(x, B(y, r)) \geq c_{2} r^{-\alpha} .
$$

As in the proof of Proposition 2.1, it follows that $\mathbf{P}_{x}\left(\tau_{B\left(x_{0}, r\right)}>t\right) \leq \exp \left(-c_{2} r^{-\alpha} t\right)$, and therefore $\mathbf{E}_{x}\left(\tau_{B\left(x_{0}, r\right)}\right) \leq c_{2}^{-1} r^{\alpha}$.

We also have the following sufficient condition for scaling properties (d) and (e).

Proposition 5.3. Assume that scaling property (a) holds. Suppose that the transition density $T_{t}(x, y)$ of a Hunt process $X_{t}$ exists, and that for some $\alpha>0, r_{0}>0$,

$$
\frac{1}{c_{[5.1]}} \min \left(t^{-n / \alpha}, \frac{t}{(d(x, y))^{n+\alpha}}\right) \leq T_{t}(x, y) \leq c_{[5.1]} \min \left(t^{-n / \alpha}, \frac{t}{(d(x, y))^{n+\alpha}}\right)
$$

for $x, y \in \mathfrak{X}$ with $d(x, y)<r_{0}$, and any $t \in\left(0, r_{0}^{\alpha}\right)$. Then Assumption $\mathrm{D}$ and scaling conditions (d) and (e) hold. The constant $c_{(2.10)}$ and the localization radius $R_{0}$ in (2.10) depend only on the constants in (5.1) (including $\alpha$ and $r_{0}$ ) and in the Ahlfors regularity condition.

Proof. Both cases $\alpha>n$ and $\alpha<n$ are very similar (in fact, slightly simpler) to the remaining case $\alpha=n$. Hence we give a detailed argument only when $\alpha=n$.

With no loss of generality we may assume that $r_{0}<$ diam $\mathfrak{X}$. We choose $k>2$ so that $m\left(B\left(x_{0}, k r\right) \backslash B\left(x_{0}, r\right)\right) \geq r^{n}$ for all $x_{0} \in \mathfrak{X}$ and $r<r_{0} / k$. Let $r<$ $r_{0} /(1+k)^{1+1 / \alpha}, x_{0} \in \mathfrak{X}, D=B\left(x_{0}, r\right)$, and let $T_{t}^{D}$ be the transition kernel of the killed process $X_{t}^{D}$. Recall that $G_{D}(x, y)=\int_{0}^{\infty} T_{t}^{D}(x, y) d t$. Let $x, y \in D$ and let $t_{1}=(d(x, y))^{\alpha}, t_{2}=(2 r)^{\alpha}$. Since $d(x, y)<2 r<r_{0}$, we have

$$
\int_{0}^{t_{1}} T_{t}^{D}(x, y) d t \leq \frac{c_{[5.1]}}{(d(x, y))^{n+\alpha}} \int_{0}^{t_{1}} t d t=\frac{c_{[5.1]}(d(x, y))^{\alpha-n}}{2},
$$

and

$$
\int_{t_{1}}^{t_{2}} T_{t}^{D}(x, y) d t \leq c_{\sqrt{5.1}} \int_{t_{1}}^{t_{2}} t^{-n / \alpha} d t=\alpha c_{(5.1)} \log \frac{2 r}{d(x, y)} .
$$

Note that for $\alpha>n$ or $\alpha<n$, we simply have a different expression for the above integral. When $t \in\left[t_{2}, 2 t_{2}\right]$, we have $t<2 t_{2}<2^{1+\alpha} r^{\alpha}<r_{0}^{\alpha}$, and hence 
$T_{t}^{D}(x, y) \leq c_{\sqrt[5.1]]{ }} t_{2}{ }^{-n / \alpha}=c_{[5.1)}(2 r)^{-n}$. Furthermore, since $T_{t_{2}} \mathbf{1} \leq \mathbf{1}$ and $d(x, z)<$ $(1+k) r<r_{0}$ for $z \in B\left(x_{0}, k r\right)$,

$$
\begin{aligned}
T_{t_{2}}^{D} \mathbf{1}(x) & \leq T_{t_{2}} \mathbf{1}_{D}(x) \leq 1-\int_{\mathfrak{X} \backslash D} T_{t_{2}}(x, z) m(d z) \\
& \leq 1-\frac{1}{c_{[5.1}} \int_{B\left(x_{0}, k r\right) \backslash B\left(x_{0}, r\right)} \frac{t_{2}}{(d(x, z))^{n+\alpha}} m(d z) \\
& \leq 1-\frac{2^{\alpha} m\left(B\left(x_{0}, k r\right) \backslash B\left(x_{0}, r\right)\right)}{c_{\text {(5.1) }}(k+1)^{n+\alpha} r^{n}} \leq 1-\frac{2^{\alpha}}{c_{\text {[5.1) }}(k+1)^{n+\alpha}} .
\end{aligned}
$$

For $s=j t_{2}+t, t \in\left[t_{2}, 2 t_{2}\right], j \geq 0$, we have $T_{s}^{D}=\left(T_{t_{2}}^{D}\right)^{j} T_{t}^{D}$. It follows that

$$
T_{s}^{D}(x, y) \leq\left(1-\frac{2^{\alpha}}{c_{[5.1)}(k+1)^{n+\alpha}}\right)^{j} \frac{c_{[5.1)}}{(2 r)^{n}}
$$

and therefore, by summing up a geometric series,

$$
\int_{t_{2}}^{\infty} T_{t}^{D}(x, y) d t \leq 2^{-\alpha}\left(c_{\underline{5.1}}\right)^{2}(k+1)^{n+\alpha} t_{2}(2 r)^{-n}=2^{-n}(k+1)^{n+\alpha}\left(c_{\sqrt[5.1]{5}}\right)^{2} r^{\alpha-n} \text {. }
$$

We conclude that $G_{D}(x, y) \leq\left(c_{[5.1)} / 2\right)+n c_{(5.1)} \log (2 r / d(x, y))+2^{-n}(k+1)^{2 n}\left(c_{[5.1)}\right)^{2}$. This gives Assumption D and property (e) Property (d) follows by simple integration.

If $X_{t}$ has $\alpha$-stable-like scaling, then, by a simple substitution, in Theorem 3.5 we have

$$
\begin{aligned}
& c_{\text {[3.9) }}\left(x_{0}, r, q, R\right) \leq c(r / R, q / R) R^{\alpha-d}, \\
& c_{\text {[3.11 }}\left(x_{0}, q, R\right) \leq c(q / R) R^{\alpha}, \\
& c_{\text {[1.1] }}\left(x_{0}, r, R\right) \leq c(r / R) .
\end{aligned}
$$

Hence the boundary Harnack inequality is uniform in all scales $R \in\left(0, R_{0}\right)$, or scaleinvariant, as claimed in Remark 3.6. We state this result as a separate theorem for future reference.

Theorem 5.4. If the assumptions of Theorem 3.5 are satisfied, and the process $X_{t}$ has $\alpha$-stable-like scaling, then the boundary Harnack inequality (BHI) is scaleinvariant: $c_{1.10}\left(x_{0}, r, R\right)$ depends only on $r / R$.

In typical applications, one verifies (typically quite straightforward) conditions (a) and (g), formula (5.1) (which has been proved for a fairly general class of processes), and condition (f)] When dealing with processes given by the Lévy kernel $\nu(x, y)$, condition (f) turns out to be the most restrictive one.

Example 5.5 (Lévy processes). Theorem 3.5 applies to a large class of Lévy processes. In this case, the notion of processes in duality and properties of the Feller generator simplify significantly; see [72].

Let $X_{t}$ be a Lévy process in $\mathfrak{X}=\mathbf{R}^{k}$ (with the Euclidean distance $d$ and Lebesgue measure $m$ ). Then $X_{t}$ is always Feller, and it is strong Feller if and only if the distribution of $X_{t}$ is absolutely continuous (with respect to the Lebesgue measure). If this is the case, Assumption $\mathrm{A}$ is satisfied: the dual of $X_{t}$ exists, and it is the reflected process, $\hat{X}_{t}-\hat{X}_{0}=-\left(X_{t}-X_{0}\right)$. Assumption $\mathrm{B}$ is always satisfied with $\mathcal{D}=C_{c}^{\infty}\left(\mathbf{R}^{k}\right)$. The Lévy kernel of $X_{t}$ is translation-invariant, $\nu(x, E)=\nu(E-x)$, where $\nu(d z)$ is the Lévy measure of $X_{t}$. Therefore, Assumption C can be restated as 
follows: the Lévy measure of $X_{t}$ is absolutely continuous, and its density function $\nu(z)$ satisfies

$$
0<c_{[2.7}^{-1} \nu\left(z_{0}\right) \leq \nu(z) \leq c_{[2.7]} \nu\left(z_{0}\right), \quad\left|z_{0}\right|>R,\left|z-z_{0}\right|<r
$$

whenever $0<r<R$, with constant $c_{(2.7)}(0, r, R)$. If, e.g., $\nu(z)$ is isotropic and radially nonincreasing, then (5.2) is equivalent to $\nu\left(z_{2}\right) \geq c \nu\left(z_{1}\right)>0$ being valid whenever $z_{1}, z_{2} \in \mathbf{R}^{k},\left|z_{1}\right| \geq 1$ and $\left|z_{2}\right|=\left|z_{1}\right|+1$. Indeed, let us assume the latter condition. By radial monotonicity, $\nu$ is locally bounded on $\mathbf{R}^{k} \backslash\{0\}$ from above and below by positive constants. Therefore, $c_{1}=c_{1}(c, \nu, r, R)>0$ exists such that $\nu\left(z_{2}\right) \geq c_{1} \nu\left(z_{1}\right)$ if $\left|z_{1}\right| \geq R-r$ and $\left|z_{2}\right|=\left|z_{1}\right|+1$. It follows that $\left(c_{1}\right)^{n} \nu\left(z_{1}\right) \leq \nu\left(z_{2}\right) \leq \nu\left(z_{1}\right)$ if $R-r \leq\left|z_{1}\right| \leq\left|z_{2}\right| \leq\left|z_{1}\right|+n$ and $n=1,2, \ldots$. Taking $n \geq r$ we obtain (5.2), as desired. Finally, Assumption D in many cases follows from estimates of the potential kernel $\mathcal{U}(x, y)=\mathcal{U}(y-x)$, or, in the recurrent case, the $\alpha$-potential kernel $\mathcal{U}_{\alpha}(x, y)=\mathcal{U}_{\alpha}(y-x)$; see Proposition 2.3.

We conclude that boundary Harnack inequality holds for a Lévy process $X_{t}$, provided that its Lévy measure satisfies (5.2), one-dimensional distributions of $X_{t}$ are absolutely continuous, and the Green functions of balls satisfy Assumption D. This class includes:

- subordinate Brownian motions which are not compound Poisson processes and have non-zero Lévy measure density function satisfying $\nu\left(z_{2}\right) \geq c \nu\left(z_{1}\right)$ if $\left|z_{1}\right| \geq 1$ and $\left|z_{2}\right|=\left|z_{1}\right|+1$ (for properties of these processes, see, e.g., [23, 64]);

- (possibly asymmetric) Lévy processes with nondegenerate Brownian part and Lévy measure satisfying (5.2);

- (possibly asymmetric) strictly stable Lévy processes, whose Lévy measure is of the form $|z|^{-d-\alpha} f(z /|z|) d z$ for a function $f$ bounded from below and above by positive constants.

Scale-invariance depends on more accurate estimates. We give here some examples and directions.

- For the class of strictly stable Lévy processes just mentioned above, scaleinvariance follows from the estimates of the transition density given in $[82$, Theorem 1.1] and Proposition [5.3, see also [28] and the references therein for related estimates in the symmetric (but anisotropic) case.

- Some Lévy processes for which Theorem 3.5 gives scale-invariant BHI are included in Example 5.6 (stable-like Lévy processes) and Example 5.8 (mixtures of isotropic stable processes, relativistic stable processes, etc.).

- A non-scale-invariant case (mixture of an isotropic stable process and the Brownian motion) is discussed in Example 5.13.

- Our results may be used to recover the recent scale-invariant BHI given in [65, Theorem 1.1]. More specifically, using our results and the first part of [65], one can obtain scale-invariant BHI for the Lévy processes considered therein (isotropic Lévy processes with the Lévy measure comparable to that of a rather general subordinate Brownian motion with some scaling properties), thus replacing the second part of [65] and significantly simplifying the whole argument of 65 .

- Similarly, the estimates given in [60, combined with Theorem 3.5] should give a compact proof of scale-invariant BHI for a class of subordinate Brownian motions with Lévy-Khintchine exponent slowly varying at $\infty$. 
Example 5.6 (Stable-like processes). Let $\mathfrak{X}$ be a closed set in $\mathbf{R}^{k}$, and let $m$ be a measure on $\mathfrak{X}$ such that $\mathfrak{X}$, with the Euclidean distance, is an Ahlfors regular $n$-space for some $n>0$. For example, $\mathfrak{X}$ can be the entire $\mathbf{R}^{k}$ or the closure of an open set in $\mathbf{R}^{k}$ (with the Lebesgue measure $m$; then $n=k$ ). On the other hand, $\mathfrak{X}$ can be a fractal set, such as Sierpiński gaskets $(n=\log (k+1) / \log 2)$ or Sierpiński carpets $\left(n=\log \left(3^{k}-1\right) / \log 3\right)$ in $\mathbf{R}^{2}$, equipped with an appropriate Hausdorff measure. By this assumption, scaling property (a) is satisfied.

Let $\alpha \in(0,2)$, and suppose that $\nu(x, y)=\nu(y, x)$ and

$$
c_{1}|x-y|^{-n-\alpha} \leq \nu(x, y) \leq c_{2}|x-y|^{-n-\alpha}, \quad x, y \in \mathfrak{X} .
$$

This immediately gives Assumption C with scaling property (g)

By [33, Theorem 1], there is a Feller, strong Feller, symmetric pure-jump Hunt process $X_{t}$ with Lévy kernel $\nu$, and the continuous transition probability $T_{t}(x, y)$ of $X_{t}$ satisfies (5.1) for some $r_{0}$. Assumption D and scaling property (e) follow by Proposition 5.3. Since $X_{t}$ is symmetric (self-dual) and has continuous transition densities, Assumption $\mathrm{A}$ is also satisfied.

Finally, we assume that Assumption B holds with scaling property (f) (see below). Under the above assumptions, scale-invariant boundary Harnack inequality holds with some localization radius. When $\mathfrak{X}$ is unbounded, $\alpha \neq n$ and scaling property (a) holds for all $r>0$, then (5.1) holds for all $t>0$ and all $x, y \in \mathfrak{X}$ (see [33]), and therefore we can take $R_{0}=\infty$.

We list some cases when Assumption B with scaling property (f) is known to hold true:

- When $\mathfrak{X}=\mathbf{R}^{k}$ and $\nu(x, y)$ is a function of $x-y$, then $X_{t}$ is a symmetric Lévy process and we can simply take $\mathcal{D}=C_{c}^{\infty}\left(\mathbf{R}^{k}\right)$.

- More generally, let $\mathfrak{X}=\mathbf{R}^{k}$, and assume that $\nu(x, y)=\kappa(x, y)|y-x|^{-k-\alpha}$ for a $C_{b}^{\infty}\left(\mathbf{R}^{k} \times \mathbf{R}^{k}\right)$ function $\kappa$. We claim that Assumption $\mathrm{B}$ with scaling property (f) holds for $\mathcal{D}=C_{c}^{\infty}\left(\mathbf{R}^{k}\right)$. Indeed, for $f \in C_{c}^{\infty}\left(\mathbf{R}^{k}\right)$ let

$$
\begin{gathered}
\tilde{\mathcal{A}} f(x)=\int_{\mathbf{R}^{k}}\left(f(x+z)-f(x)-\frac{z}{1+|z|^{2}} \cdot \nabla f(x)\right) \frac{\kappa(x, x+z)}{|z|^{k+\alpha}} d z \\
+\left(\int_{\mathbf{R}^{k}} \frac{z}{1+|z|^{2}} \frac{\kappa(x, x+z)-\kappa(x, x)}{|z|^{k+\alpha}} d z\right) \cdot \nabla f(x) .
\end{gathered}
$$

Then $\tilde{\mathcal{A}}$ is a symmetric pseudo-differential operator with appropriately smooth symbol, and by [50, Theorem 5.7], the closure of $\tilde{\mathcal{A}}$ is the Feller generator of a symmetric Hunt process $\tilde{X}_{t}$ (we omit the details). Since the pure-jump Feller processes $X_{t}$ and $\tilde{X}_{t}$ have equal Lévy kernels, they are in fact equal processes, and hence the closure of $\tilde{\mathcal{A}}$ is the Feller generator of $X_{t}$. Assumption $\mathrm{B}$ with $\mathcal{D}=C_{c}^{\infty}\left(\mathbf{R}^{k}\right)$ follows, and scaling property (f) is a simple consequence of (5.4). See also [49,79].

- When $\alpha \in(0,1), \mathfrak{X}$ is the closure of an open Lipschitz set, and $\nu(x, y)=$ $c|x-y|^{-k-\alpha}$, then the desired condition is satisfied by $\mathcal{D}=C_{c}^{\infty}\left(\mathbf{R}^{k}\right)$ (see [45, Theorem 6.1(i)]).

- When $\alpha \in[1,2), \mathfrak{X}$ is the closure of an open set with $C^{1, \beta}$-smooth boundary for some $\beta>\alpha-1$, and $\nu(x, y)=c|x-y|^{-k-\alpha}$, then one can take $\mathcal{D}$ to be the class of $C_{c}^{\infty}\left(\mathbf{R}^{k}\right)$ functions with normal derivative vanishing everywhere on the boundary of $\mathfrak{X}$ (see [45, Theorem 6.1(ii)]). 
- For the case when $X_{t}$ is a subordinate diffusion on $\mathfrak{X}$, see Example 5.7. In this case, when $\mathfrak{X}$ is a fractal set, one can even deal with $\alpha$ greater than 2 .

Note that an analytical proof of Theorem 3.4 discussed in Remark 4.12 may lead to a generalization of this example, which would not require Assumption B.

Example 5.7 (Stable-like subordinate diffusions in metric measure spaces). Suppose that $(\mathfrak{X}, d, m)$ is an Ahlfors regular $n$-space for some $n>0$. Assume that the metric $d$ is uniformly equivalent to the shortest-path metric in $\mathfrak{X}$. Suppose that there is a diffusion process $Z_{t}$ with a symmetric, continuous transition density $T_{t}^{Z}(x, y)$ satisfying the sub-Gaussian bounds

$$
\begin{gathered}
\frac{c_{1}}{t^{n / d_{w}}} \exp \left(-c_{2}\left(\frac{d(x, y)^{d_{w}}}{t}\right)^{1 /\left(d_{w}-1\right)}\right) \leq T_{t}^{Z}(x, y) \\
\leq \frac{c_{3}}{t^{n / d_{w}}} \exp \left(-c_{4}\left(\frac{d(x, y)^{d_{w}}}{t}\right)^{1 /\left(d_{w}-1\right)}\right)
\end{gathered}
$$

for all $x, y \in \mathfrak{X}$ and $t \in\left(0, t_{0}\right)\left(t_{0}=\infty\right.$ when $\mathfrak{X}$ is unbounded). Here $d_{w} \geq 2$ is the walk dimension of the space $\mathfrak{X}$. The existence of such a diffusion process $Z_{t}$ is well-known when $\mathfrak{X}$ is a Riemannian manifold $\left(d_{w}=2\right.$; see 43 ]), the $k$ dimensional Sierpiński gasket $\left(d_{w}=\log (k+3) / \log 2>2\right.$; see [11]), more general nested fractals [41,68], or the Sierpiński carpets [7,8]; see [59] for more information.

Let $\alpha \in\left(0, d_{w}\right)$ and let $X_{t}$ be the stable-like process obtained by subordination of $Z_{t}$ with the $\alpha / d_{w}$-stable subordinator $\eta_{t}, X_{t}=Z\left(\eta_{t}\right)$. These processes were first studied in $[27,69,76]$. By the subordination formula, the transition density estimate (5.1) holds for some $r_{0}$ (if $\mathfrak{X}$ is unbounded, then it was proved in [27] that we can take $r_{0}=\infty$ ).

Since $X_{t}$ is symmetric and has continuous transition densities, Assumption A is clearly satisfied. The Lévy kernel of $X_{t}$ satisfies $c^{-1} d(x, y)^{-n-\alpha} \leq \nu(x, y) \leq$ $c d(x, y)^{-n-\alpha}$ (see [27]), and Assumption C with scaling property (g) follows. Assumption $\mathrm{D}$ and scaling property (e) follow from the transition density estimate (5.1) by Proposition 5.3, see also [27, Lemmas 5.3 and 5.6]. Finally, Assumption B with scaling property[(f) follows by the construction of [71, Section 2]. Roughly speaking, the method of [71] yields smooth bump functions in the domain of the generator of the diffusion $Z_{t}$ with appropriate scaling. By the subordination formula, these bump functions are in the domain of $\mathcal{A}$, and the constants scale appropriately. Since there are some nontrivial issues related to the construction, we repeat the construction with all details in Appendix A. By Corollary A.4 there, Assumption $\mathrm{B}$ is satisfied with scaling property $(\mathrm{f})$

We conclude that scale-invariant boundary Harnack inequality for $X_{t}$ holds in the full range of $\alpha \in\left(0, d_{w}\right)$. Noteworthy, we obtain a regularity result also for $\alpha \geq 2$, when Lipshitz functions no longer belong to the domain of the Dirichlet form of $X_{t}$.

This example can be extended in various directions. Instead of taking $\eta_{t}$ to be the $\alpha / d_{w}$-stable subordinator, one can consider a subordinator $\eta_{t}$ whose Laplace exponent $\psi$ is a complete Bernstein function regularly varying of order $\alpha / d_{w}$ ( $\alpha \in$ $\left.\left(0, d_{w}\right)\right)$ at infinity. Such subordinators have zero drift, and they have Lévy measures with completely monotone density functions which are regularly varying of order $-1-\alpha / d_{w}$ at 0 . Their potential kernel is regularly varying of order $-1+\alpha / d_{w}$ at 0 . We refer the reader to $23,64,73$ for more information about subordination, 
complete Bernstein functions and regular variation. By the subordination formula, following the method applied for the Euclidean case $\mathfrak{X}=\mathbf{R}^{k}$ in 64,65 , one can obtain two-sided estimates for the Lévy kernel $\nu(x, y)$ and the potential kernel $\mathcal{U}(x, y)$ in terms of $\psi$, at least when $\mathfrak{X}$ is unbounded and $\alpha<d$. These estimates are sufficient to prove the scale-invariant boundary Harnack inequality.

Similar methods should be applicable also when $X_{t}$ is recurrent (that is, $\mathfrak{X}$ is bounded, or $\alpha \geq d)$. In this case, estimates of $\mathcal{U}(x, y)$ need to be replaced by estimates of the $\lambda$-potential kernel $\mathcal{U}_{\lambda}(x, y)$. Other interesting directions are the case of slowly varying $\psi$, which corresponds to $\alpha=0$, and, on the other hand, the case of pure-jump processes with $\psi$ regularly varying of order 1 (that is, $\alpha=d_{w}$ ). Finally, one can perturb processes considered above, in a similar way as in the next example.

Example 5.8 (Stability under small perturbations). Let $\mathfrak{X}=\mathbf{R}^{k}, d$ be the Euclidean distance, $m$ be the Lebesgue measure, and $\alpha \in(0,2)$. Suppose that $\tilde{\nu}(x, y)$ is a Lévy kernel of a Hunt process $\tilde{X}_{t}$ considered in Example [5.6, and $\tilde{\mathcal{A}}$ is the corresponding Feller generator. For example, $\tilde{\nu}(x, y)$ can be any function of $y-x$ satisfying (5.3). In this example we consider a perturbation $\nu(x, y)$ of the kernel $\tilde{\nu}(x, y)$.

Although a more general construction is feasible, we are satisfied with the following setting. Let $\nu(x, y)=\tilde{\nu}(x, y)+n(x, y)$, where $n(x, y)$ is chosen so that $\nu(x, y)$ satisfies the scaling property $(\mathrm{g}), n(x, y)$ and $\hat{n}(x, y)=n(y, x)$ are kernels of bounded operators on $C_{0}\left(\mathbf{R}^{k}\right)$, and

$$
\int_{\mathbf{R}^{k}} n(x, y) d y=\int_{\mathbf{R}^{k}} \hat{n}(x, y) d y, \quad x \in \mathbf{R}^{k}
$$

the last assumption guarantees that $m$ is an excessive (in fact, invariant) measure for the process $X_{t}$ defined below.

The formula $\mathcal{N} f(x)=\int_{\mathbf{R}^{k}}(f(y)-f(x)) n(x, y) d y$ defines a bounded linear operator on $C_{0}\left(\mathbf{R}^{k}\right)$, and $\mathcal{A}=\tilde{\mathcal{A}}+\mathcal{N}$ (defined on the domain of $\tilde{\mathcal{A}}$ ) has the positive maximum property. By a standard perturbation argument, $\mathcal{A}$ is the Feller generator of a Hunt process $X_{t}$, and $\nu(x, y)$ is the Lévy kernel of $X_{t}$. The process $\hat{X}_{t}$ and its Feller generator $\hat{\mathcal{A}}$ are constructed in a similar manner, using the Feller generator of the dual of $\tilde{X}_{t}$ and the kernel $\hat{n}(x, y)$. It is easy to see that $\int_{\mathbf{R}^{k}} \mathcal{A} f(x) g(x) d x=\int_{\mathbf{R}^{k}} f(x) \hat{\mathcal{A}} g(x) d x$ for $f, g \in C_{c}^{\infty}\left(\mathbf{R}^{k}\right)$, from which it follows that $\hat{X}_{t}$ is indeed the dual of $X_{t}$.

The transition density of $\tilde{X}_{t}$ satisfies (5.1) (see Example 5.6). The process $X_{t}$ can be constructed probabilistically using $\tilde{X}_{t}$ and Meyer's method of adding and removing jumps. Hence, by [9, Lemma 3.6] and [10, Lemma 3.1(c)], the transition density of $X_{t}$ exists and also satisfies (5.1) for smaller $r_{0}$ (see also 30, Proposition 2.1]).

It follows that Assumption $\mathrm{A}$ is satisfied. Assumption $\mathrm{B}$ holds with $\mathcal{D}=$ $C_{c}^{\infty}\left(\mathbf{R}^{k}\right)$, and scaling property (f) (with finite $R_{0}$ ) follows from the $\alpha$-stable-like scaling of $\tilde{\mathcal{A}}$ and boundedness of $\mathcal{N}$. Since we assumed that (g) holds true, Assumption $\mathrm{C}$ is satisfied with scaling properties (b), (c) Assumption D and scaling properties (d), (e) follow from transition density estimate (5.1) by Proposition 5.3. Hence, scale-invariant boundary Harnack inequality holds true for $X_{t}$.

The above setting includes mixtures of isotropic stable processes (Lévy processes generated by $\mathcal{A}=-(-\Delta)^{\alpha / 2}-c(-\Delta)^{\beta / 2}$ with $0<\beta<\alpha<2$ and $c>0$ ) and 
relativistic stable processes (Lévy processes generated by $\mathcal{A}=m-\left(-\Delta+m^{2 / \alpha}\right)^{\alpha / 2}$ with $m>0)$. Also, the dependence of constants on the parameters $c, \beta, m$ can be easily tracked. Since the perturbation $n(x, y)$ can be asymmetric, many nonsymmetric processes are included. Finally, this example can be adapted to the setting of Ahlfors regular $n$-sets in $\mathbf{R}^{k}$, as in Example [5.6.

Example 5.9 (Processes killed by a Schrödinger potential). Suppose that the assumptions for the boundary Harnack inequality in Theorem 3.5 are satisfied. Let $\mathfrak{X}^{\prime}$ be an open set in $\mathfrak{X}$. Let $M_{t}$ be a strong right-continuous multiplicative functional quasi-left-continuous on $[0, \infty)$, for which all points of $\mathfrak{X}^{\prime}$ are permanent, and such that $M_{t}=0$ for $t \geq \tau_{\mathfrak{X}^{\prime}}$. Finally, let $X_{t}^{M}$ be the subprocess corresponding to $M_{t}$ (in a similar way as in Section 4] see [18] for definitions). Then $X_{t}^{M}$ is a Hunt process on $\mathfrak{X}^{\prime}$, uniquely determined by the relation $\mathbf{P}_{x}^{M}\left(X_{t}^{M} \in E\right)=\mathbf{E}_{x}\left(M_{t} ; X_{t} \in E\right)$ for any $E \subseteq \mathfrak{X}^{\prime}$ and $x \in \mathfrak{X}^{\prime}$.

Assume that $M_{t}$ is a continuous function of $t \in\left[0, \tau_{\mathfrak{X}^{\prime}}\right)$. We claim that in this case the Lévy kernel $\nu^{M}(x, y)$ of $X_{t}^{M}$ is again given by $\nu(x, y)$, restricted to $\mathfrak{X}^{\prime} \times \mathfrak{X}^{\prime}$. Indeed, by formula (4.6) of Lemma 4.3. for $x \in \mathfrak{X}^{\prime}$ and $f \in \mathcal{D}(\mathcal{A})$ vanishing in a neighborhood of $x$, we have

$$
\begin{aligned}
\mathbf{E}_{x}^{M}\left(f\left(X_{t}^{M}\right)\right)-f(x) & =\mathbf{E}_{x}\left(f\left(X_{t}\right) M_{t}\right)-f(x) \\
& =\mathbf{E}_{x}\left(\int_{0}^{t} \mathcal{A} f\left(X_{t}\right) M_{t} d t\right)+\mathbf{E}_{x}\left(\int_{0}^{t} f\left(X_{t}\right) d M_{t}\right) .
\end{aligned}
$$

When divided by $t$, this converges (for a fixed $x$ ) to $\mathcal{A} f(x)$ as $t \rightarrow 0^{+}$. Hence, $\nu^{M} f(x)=\nu f(x)$. By an approximation argument, this holds for any $f \in C_{c}\left(\mathfrak{X}^{\prime}\right)$ vanishing in a neighborhood of $x$, proving our claim. (Note that, however, in general, functions in $\mathcal{D}(\mathcal{A})$ need not belong to the domain of the generator of $X_{t}^{M}$, even if $\mathfrak{X}^{\prime}=\mathfrak{X}$.)

We remark that many such functionals $M_{t}$ are related to Schrödinger potentials $V$ : for a nonnegative function $V$, we have $M_{t}=\exp \left(-\int_{0}^{t} V\left(X_{s}\right) d s\right)$ for $t<\tau_{\mathfrak{X}^{\prime}}$; see [18. A similar construction was used in Section 4 for a particular choice of $V$. In some applications, the potential $V$ can take negative values, the case not covered by this example.

Let $D \subseteq \mathfrak{X}$ be an open set. By the definition of a subharmonic function, a nonnegative function $f$ regular subharmonic on $D \cap \mathfrak{X}^{\prime}$ with respect to the process $X_{t}^{M}$, extended by $f(x)=0$ for $x \in \mathfrak{X} \backslash \mathfrak{X}^{\prime}$, is also regular subharmonic in $D$ with respect to $X_{t}$. Hence, the hypothesis of Theorem 3.4 holds for $X_{t}^{M}$ with the same constant $c_{(3.9)}$. Of course, one needs to replace the sets in the statement of Theorem 3.4 by their intersections with $\mathfrak{X}^{\prime}$.

We also claim that Lemma 3.1 holds for $X_{t}^{M}$ with the same constant. Indeed, with the definitions of the proof of Lemma 3.1 and $D^{\prime}=D \cap \mathfrak{X}^{\prime}$, for $x \in B\left(x_{0}, r\right) \cap \mathfrak{X}^{\prime}$ we have

$$
\begin{aligned}
\mathbf{P}_{x}^{M}\left(X_{\tau_{D^{\prime}}}^{M} \in \mathfrak{X}^{\prime} \backslash B\left(x_{0}, R\right)\right) & =\mathbf{E}_{x}\left(M_{\tau_{D}} ; X_{\tau_{D}} \in \mathfrak{X} \backslash B\left(x_{0}, R\right)\right) \\
& \leq \mathbf{E}_{x}\left(f\left(X_{\tau_{D}}\right) M_{\tau_{D}}\right)-f(x) .
\end{aligned}
$$

By formula (4.6) of Lemma 4.3.

$$
\mathbf{E}_{x}\left(f\left(X_{\tau_{D}}\right) M_{\tau_{D}}\right)-f(x)=\mathbf{E}_{x}\left(\int_{0}^{\tau_{D}} \mathcal{A} f\left(X_{t}\right) M_{t} d t\right)+\mathbf{E}_{x}\left(\int_{\left[0, \tau_{D}+\right]} f\left(X_{t}\right) d M_{t}\right) .
$$


The second summand on the right hand side is nonpositive. It follows that

$$
\begin{aligned}
\mathbf{P}_{x}^{M}\left(X_{\tau_{D}}^{M} \in \mathfrak{X}^{\prime} \backslash B\left(x_{0}, R\right)\right) & \leq \mathbf{E}_{x}\left(\int_{0}^{\tau_{D}} M_{t} d t\right) \sup _{y \in \mathfrak{X}} \mathcal{A} f(y) \\
& =\mathbf{E}_{x}^{M}\left(\tau_{D^{\prime}}\right) \sup _{y \in \mathfrak{X}} \mathcal{A} f(y),
\end{aligned}
$$

as desired.

In Lemma 3.2, only the estimates of the Lévy measure and mean exit time are used. Therefore, Lemma 3.2 also holds for the process $X_{t}^{M}$ with unaltered constants. In a similar way, the proof of Theorem 3.5 works for the process $X_{t}^{M}$ without modifications. We conclude that the boundary Harnack inequality holds for $X_{t}^{M}$ with the same constants. For convenience, we state this result as a separate theorem.

Theorem 5.10. Suppose that Assumptions [A, B, [ and D] hold true. Let $\mathfrak{X}^{\prime}$ be an open subset of $\mathfrak{X}$, and let $X_{t}^{M}$ be a subprocess of $X_{t}$, with state space $\mathfrak{X}^{\prime}$, corresponding to a strong right-continuous multiplicative functional for $X_{t}$, continuous before $X_{t}$ hits $\mathfrak{X} \backslash \mathfrak{X}^{\prime}$, vanishing after that time, and quasi-left continuous on $[0, \infty)$. Then the boundary Harnack inequality holds true for the process $X_{t}^{M}$ with the same constant $c_{(1.1)}$ given by (3.10). More precisely, if $x_{0} \in \mathfrak{X}, 0<r<R<R_{0}$, $D \subseteq B\left(x_{0}, R\right)$ is open, $f, g$ are nonnegative regular harmonic functions in $D \cap \mathfrak{X}^{\prime}$ (with respect to the process $\left.X_{t}^{M}\right)$, and $f, g$ vanish in $\left(B\left(x_{0}, R\right) \backslash D\right) \cap \mathfrak{X}^{\prime}$, then we have

$$
f(x) g(y) \leq c_{\text {1.1] }} g(x) f(y), \quad x, y \in B\left(x_{0}, r\right) \cap D \cap \mathfrak{X}^{\prime},
$$

where $c_{[1.1)}=c_{[1.1)}\left(x_{0}, r, R\right)$ does not depend on $M_{t}$.

We remark that the continuity assumption for $M_{t}$ is essential. If, for example, $M_{t}$ is equal to 1 until the first jump larger than 1, and then 0 , the boundary Harnack inequality typically does not hold, by an argument similar to one in Example 5.14 below.

Example 5.11 (Actively reflected and censored stable processes). Let $\mathfrak{X}^{\prime} \subseteq \mathbf{R}^{k}$ be open and let $\mathfrak{X}$ be the closure of $\mathfrak{X}^{\prime}$ in $\mathbf{R}^{k}$. Suppose that $\mathfrak{X}$ satisfies property (a), Let $\nu(x, y)=c|x-y|^{-n-\alpha}$. As in Example [5.6] under suitable assumptions on $\mathfrak{X}$, there is a stable-like process $X_{t}$ with the Lévy kernel $\nu(x, y)$, and scale-invariant BHI holds for $X_{t}$. In [21, the process $X_{t}$ is called an actively reflected $\alpha$-stable process in $\mathfrak{X}$, and the process $X_{t}^{\prime}$, obtained from $X_{t}$ by killing it upon hitting $\mathfrak{X} \backslash \mathfrak{X}^{\prime}$, is called a censored $\alpha$-stable process in $\mathfrak{X}^{\prime}$ (see [21, Remark 2.1]). Clearly, the boundary Harnack inequality for $X_{t}^{\prime}$ is the special case of the boundary Harnack inequality for $X_{t}$, corresponding to open sets $D$ contained in $\mathfrak{X}^{\prime}$. (Note that this is in fact a special case of Theorem 5.10, with $M_{t}=1$ for $t<\tau_{\mathfrak{X}^{\prime}}$.) Hence, we have scale-invariant BHI for the actively reflected $\alpha$-stable process $X_{t}$ and the censored $\alpha$-stable process $X_{t}^{\prime}$, whenever $\mathfrak{X}^{\prime}$ is a Lipschitz set in the case $\alpha \in(0,1)$, and $\mathfrak{X}^{\prime}$ is an open set with $C^{1, \beta}$-smooth boundary for some $\beta>\alpha-1$ in the case $\alpha \in[1,2)$. The above extends the results of [21,44].

Example 5.12 (Gradient-type perturbations of stable processes). Let $\alpha \in(1,2)$. If $b: \mathbf{R}^{k} \rightarrow \mathbf{R}^{k}$ is bounded and differentiable, partial derivatives of $b$ are bounded, and $\operatorname{div} b=0$, then the process $X_{t}$ generated by $-(-\Delta)^{\alpha / 2}+b \cdot \nabla$ and the process $\hat{X}_{t}$ generated by $-(-\Delta)^{\alpha / 2}-b \cdot \nabla$ are mutually dual. Such processes are considered 
in the recent paper [53. The Lévy kernels of $X_{t}$ and $\hat{X}_{t}$ are the same as that of the isotropic $\alpha$-stable Lévy process generated by $(-\Delta)^{\alpha / 2}$; see [25]. Furthermore, $\mathcal{D}=C_{c}^{\infty}\left(\mathbf{R}^{k}\right)$ is contained in the domains of $\mathcal{A}$ and $\hat{\mathcal{A}}$. Therefore, a scale-invariant (with finite $R_{0}$ ) boundary Harnack inequality holds for the process $X_{t}$.

We conclude this article with some negative or partially negative examples.

Example 5.13 (Lévy processes with Brownian component). Let $\mathfrak{X}=\mathbf{R}^{k}, d$ be the Euclidean distance, $m$ be the Lebesgue measure, and $\alpha \in(0,2)$. Let $X_{t}$ be the sum of two independent processes, the Brownian motion and the isotropic $\alpha$-stable Lévy process. That is, $X_{t}$ is the Lévy process with generator $\mathcal{A}=c_{1} \Delta-c_{2}(-\Delta)^{\alpha / 2}$.

Clearly, $X_{t}$ is symmetric and has transition densities, so Assumption $\mathrm{A}$ is satisfied. Furthermore, $\mathcal{D}(\mathcal{A})$ contains $C_{c}^{\infty}\left(\mathbf{R}^{k}\right)$, and hence Assumption $\mathrm{B}$ is satisfied with 2-stable-like scaling: the property (f) holds with $\alpha$ replaced by 2 . On the other hand, Assumption C clearly holds with $\alpha$-stable-like scaling (g), Furthermore, detailed estimates for the transition density of $X_{t}$ can be established (34]), from which Assumption $\mathrm{D}$ follows as in Proposition 5.3, with 2-stable scaling.

It follows that boundary Harnack inequality holds despite the diffusion component. However, the constant $c_{1.1]}\left(x_{0}, r, R\right)$ is not bounded when, for example, $R=2 r$ and $r \rightarrow 0^{+}$. This is typical behavior for processes comprising both jump and diffusion parts, and for general open sets one cannot expect a scale-invariant result: the boundary Harnack inequality in the form given in (BHI) does not hold for the Brownian motion without some regularity assumptions on the boundary of $D$; cf. [14]. On the other hand, the scale-invariant boundary Harnack inequality for $X_{t}$ in more smooth domains was established in 32 .

Example 5.14 (Truncated stable processes). This example shows why Assumption $\mathrm{C}$ is essential for the boundary Harnack inequality in the form given in (BHI). Consider the truncated isotropic $\alpha$-stable Lévy process $X_{t}$ in $\mathfrak{X}=\mathbf{R}^{k}, \alpha \in(0,2)$, $n \geq 1$. This is a pure-jump Lévy process with Lévy kernel

$$
\nu(x, y)=c|x-y|^{-n-\alpha} \mathbf{1}_{B(x, 1)}(y) .
$$

Clearly, Assumptions $\mathrm{A}, \mathrm{B}$ and D, as well as formula (2.9), hold true with $\alpha$-stablelike scaling and $R_{0}=1$, but Assumption $\mathrm{C}$ is violated.

We examine two specific harmonic functions. Let $v$ be a vector in $\mathbf{R}^{d}$ with $|v|=2 / 3$, let $r \in(0,1 / 6)$ be a small number, and define $B_{1}=B\left(x_{1}, r\right)$ and $B_{2}=B\left(x_{2}, r\right)$, where $x_{1}, x_{2} \in \mathbf{R}^{k}$ are arbitrary points satisfying $x_{1}-x_{2}=v$. Let $D=B_{1} \cup B_{2}, E_{1}=B_{1}+v, E_{2}=B_{2}-v$, and let $f_{j}(x)=\mathbf{P}_{x}\left(X\left(\tau_{D}\right) \in E_{j}\right)$. Suppose that $x \in B_{1}$. By (2.12), we have

$$
3^{-n-\alpha} c\left|E_{1}\right| \mathbf{E}_{x} \tau_{B_{1}} \leq f_{1}(x) \leq 3^{n+\alpha} c\left|E_{1}\right| \mathbf{E}_{x} \tau_{D} .
$$

When $x \in B_{2}$, then, again by (2.12),

$$
\begin{aligned}
f_{1}(x) & \leq \mathbf{P}_{x}\left(X\left(\tau_{B_{2}}\right) \in B_{1}\right) \cdot \sup _{y \in B_{1}} f_{1}(y) \\
& \leq c 3^{n+\alpha}\left|B_{1}\right| \mathbf{E}_{x} \tau_{B_{2}} \cdot 3^{n+\alpha} c\left|E_{1}\right| \sup _{y \in B_{2}} \mathbf{E}_{y} \tau_{D} .
\end{aligned}
$$


Similar estimates hold true for $f_{2}$. It follows that

$$
\begin{aligned}
\frac{f_{1}\left(x_{2}\right) f_{2}\left(x_{1}\right)}{f_{1}\left(x_{1}\right) f_{2}\left(x_{2}\right)} & \leq c_{n, \alpha}^{2}\left(3^{n+\alpha}\right)^{6}\left|B_{1}\right|\left|B_{2}\right|\left(\sup _{y \in D} \mathbf{E}_{y} \tau_{D}\right)^{2} \\
& \leq c_{n, \alpha}^{2}\left(3^{n+\alpha}\right)^{6}|B(0,1)|^{2} r^{2 n}\left(\sup _{y \in B(0,1)} \mathbf{E}_{y} \tau_{B(0,1)}\right)^{2} .
\end{aligned}
$$

This ratio can be arbitrarily small when $r \rightarrow 0$, and therefore (BHI) cannot hold for the truncated stable process uniformly with respect to the domain. We remark that by an appropriate modification of the above example, one can even construct a single domain (an infinite union of balls) for which (BHI) is false. Also, modifications of the above example for other truncated processes or for processes with super-exponential decay of the density of the Lévy measure can be given.

On the other hand, if the regular harmonic functions $f$ and $g$ (of the truncated $\alpha$-stable process $X_{t}$ ) vanish outside a unit ball, then clearly $f$ and $g$ are harmonic in $D$ also with respect to the standard (that is, nontruncated) isotropic $\alpha$-stable process in $\mathbf{R}^{k}$. Therefore, the boundary Harnack inequality actually holds true for such functions. A different version of boundary Harnack inequality was proved for $X_{t}$ under some regularity assumptions on the domain of harmonicity in [61,62].

\section{Appendix A. Smooth bump functions on metric measure spaces WITH SUB-GAUSSIAN HEAT KERNELS}

In this part we repeat the construction of smooth bump functions of 71 . We adopt the setting of Example 5.7. $Z_{t}$ is a diffusion process on an Ahlfors regular $n$-space $\mathfrak{X}$, the transition semigroup $T_{t}^{Z}$ of $Z_{t}$ satisfies sub-Gaussian bounds (5.5), and $X_{t}$ is defined to be the process $Z_{t}$ subordinated by an independent $\alpha / d_{w}$-stable subordinator, $\alpha \in\left(0, d_{w}\right)$. The generator of $Z_{t}$ serves as the (Neumann) Laplacian $\Delta$ on $\mathfrak{X}$, and $T_{t}^{Z}$ is the heat semigroup.

Let $h=T_{t}^{Z} g$ for some $t>0$ and $g \in L^{2}(\mathfrak{X})$. One of the main results of [7], Theorem 2.2, states that given any compact $K$ and $\varepsilon>0$, there is a function $f$ such that $f \in \mathcal{D}\left(\Delta^{l}\right)$ for all $l>0, f(x)=h(x)$ on $K$ and $f(x)=0$ when $\operatorname{dist}(x, K) \geq \varepsilon$. There are at least three issues when one tries to apply this result in our setting.

First, Theorem 2.2 in 71 is given under the assumption that the spectral gap of $\Delta$ is positive. However, this assumption is used only in the proof of Lemma 2.6, which contains a flaw: positivity of the spectral gap $\lambda$ does not imply the inequality $\left\|P_{t} f-f\right\|_{L^{2}(\mathfrak{X})} \leq \lambda t\|f\|_{L^{2}(\mathfrak{X})}$ (see line 3 on page 1769 and line 12 on page 1773 in [71]). This issue has been resolved by the authors of [71] in an unpublished note, containing a corrected version of the proof of Lemma 2.6. The new argument does not involve the condition on the spectral gap, which therefore turns out to be superfluous. For future reference, we provide the corrected version of the proof of Lemma 2.6 below.

Second, to get Assumption $B$, we need to apply the above theorem with $h(x)=1$ for $x \in K$, where $h=T_{t}^{Z} g$. This condition is satisfied when $g(x)=1$ for all $x \in \mathfrak{X}$. However, such a function $g$ is in $L^{2}(\mathfrak{X})$ only when $m$ is a finite measure, and the general case is not covered by [71. For that reason, we choose to repeat the construction of 71 in the $L^{\infty}(\mathfrak{X})$ (instead of the $L^{2}(\mathfrak{X})$ ) setting.

Finally, for a scale-invariant boundary Harnack inequality, we need an upper bound for $\|\Delta f\|_{L^{\infty}(\mathfrak{X})}$ with explicit dependence on scale, that is, explicit in $\varepsilon$ and 
the size (e.g. the diameter) of $K$. Such properties of the estimates are irrelevant in [71, but it turns out that they can be obtained by carefully following the proof of Theorem 2.2 in 71 .

For the above reasons, we decide to give a complete proof of an $L^{\infty}(\mathfrak{X})$ version of Theorem 2.2 in [71. However, it should be emphasized that this method was completely developed in [71. Although we only need the result for $g(x)=h(x)=1$ for all $x \in \mathfrak{X}$, for future reference we consider the general case.

Theorem A.1 (A variant of [71, Theorem 2.2]). Suppose that $K \subseteq \mathfrak{X}$ is a compact set, $\varepsilon, s>0$ and $h=T_{s}^{Z} g$ for some $g \in L^{\infty}(\mathfrak{X})$. Then there is a function $f \in L^{\infty}(\mathfrak{X})$ such that $f(x)=h(x)$ for $x \in K, f(x)=0$ when $\operatorname{dist}(x, K) \geq \varepsilon$, and $f \in \mathcal{D}\left(\Delta^{l}\right)$ for any $l>0$. Furthermore, the $L^{\infty}(\mathfrak{X})$ norm of $f$ is bounded by the $L^{\infty}(\mathfrak{X})$ norm of $g, f$ is nonnegative if $g$ is nonnegative, and for all $l>0$ we have

$$
\left\|\Delta^{l} f\right\|_{L^{\infty}(\mathfrak{X})} \leq \frac{c_{\text {A.1) }}(\operatorname{diam} K+\varepsilon)^{n / 2}}{\varepsilon^{l d_{w}+n / 2}}\|g\|_{L^{\infty}(\mathfrak{X})},
$$

where $c_{\underline{\mathrm{A} .1}}=c_{\underline{\mathrm{A} .1 \mathrm{~B}}}\left(l, \varepsilon^{d_{w}} / s, Z_{t}\right)$.

Proof. We divide the argument into five steps. All constants in this proof may depend not only on the parameters given in parentheses, but also on the space $\mathfrak{X}$ and the process $Z_{t}$. Since we never refer to the semigroup of the subordinate process $X_{t}$, in this proof for simplicity we write $T_{t}=T_{t}^{Z}$. Furthermore, also in this proof only, we extend $\Delta$ to the $L^{\infty}(\mathfrak{X})$ generator of $T_{t}$ (recall that originally $\Delta$ was defined as the $C_{0}(\mathfrak{X})$ generator), and denote by $\Delta_{L^{2}(\mathfrak{X})}$ the $L^{2}(\mathfrak{X})$ generator of $T_{t}$, that is, the generator of the semigroup of operators $T_{t}$ acting on $L^{2}(\mathfrak{X})$. Clearly, $\Delta f=\Delta_{L^{2}(\mathfrak{X})} f m$-a.e. whenever $f \in \mathcal{D}(\Delta) \cap \mathcal{D}\left(\Delta_{L^{2}(\mathfrak{X})}\right)$.

Step 1. We begin with some general estimates. By the spectral theorem and the inequality $\lambda^{l} e^{-\lambda t} \leq(l e / t)^{l}$, for any $f \in L^{2}(\mathfrak{X})$ and $l \geq 0$, we have $T_{t} f \in \mathcal{D}\left(\left(\Delta_{L^{2}(\mathfrak{X})}\right)^{l}\right)$, and

$$
\left\|\left(\Delta_{L^{2}(\mathfrak{X})}\right)^{l} T_{t} f\right\|_{L^{2}(\mathfrak{X})} \leq(l e / t)^{l}\|f\|_{L^{2}(\mathfrak{X})} .
$$

Furthermore, by sub-Gaussian estimates (5.5),$\left\|T_{t}(x, \cdot)\right\|_{L^{2}(\mathfrak{X})}=\left(T_{2 t}(x, x)\right)^{1 / 2} \leq$ $c_{1} t^{-n /\left(2 d_{w}\right)}$. Hence,

$$
\left\|T_{t} f\right\|_{L^{\infty}(\mathfrak{X})} \leq c_{1} t^{-n /\left(2 d_{w}\right)}\|f\|_{L^{2}(\mathfrak{X})} .
$$

We find that

$$
\begin{aligned}
& \left\|\frac{T_{s} T_{t} f-T_{t} f}{s}-\Delta_{L^{2}(\mathfrak{X})} T_{t} f\right\|_{L^{\infty}(\mathfrak{X})} \\
& \quad \leq \frac{c_{1}}{(t / 2)^{n /\left(2 d_{w}\right)}}\left\|\frac{T_{s} T_{t / 2} f-T_{t / 2} f}{s}-\Delta_{L^{2}(\mathfrak{X})} T_{t / 2} f\right\|_{L^{2}(\mathfrak{X})} \rightarrow 0
\end{aligned}
$$

as $s \rightarrow 0^{+}$. It follows that $T_{t} f \in \mathcal{D}(\Delta)$, with $\Delta T_{t} f=\Delta_{L^{2}(\mathfrak{X})} T_{t} f$. By a similar argument, $T_{t} f \in \mathcal{D}\left(\Delta^{l}\right)$ for any $l \geq 0$, and

$$
\begin{aligned}
\left\|\Delta^{l} T_{t} f\right\|_{L^{\infty}(\mathfrak{X})}= & \left\|T_{t / 2} \Delta^{l} T_{t / 2} f\right\|_{L^{\infty}(\mathfrak{X})} \leq \frac{c_{2}}{t^{n /\left(2 d_{w}\right)}}\left\|\Delta^{l} T_{t / 2} f\right\|_{L^{2}(\mathfrak{X})} \\
& \leq \frac{c_{3}(l)}{t^{l+n /\left(2 d_{w}\right)}}\|f\|_{L^{2}(\mathfrak{X})} .
\end{aligned}
$$


Sub-Gaussian estimate (5.5) and Ahlfors regularity of $\mathfrak{X}$ also give the following estimate: for any set $E \subseteq \mathfrak{X}$, any $\varepsilon>0$ and any $f \in L^{\infty}(\mathfrak{X})$ or $f \in L^{1}(\mathfrak{X})$ vanishing in the $\varepsilon$-neighborhood of $E$, we have

$$
\left\|T_{t} f\right\|_{L^{\infty}(E)} \leq D(\varepsilon, t)\|f\|_{L^{\infty}(\mathfrak{X})}, \quad \text { and } \quad\left\|T_{t} f\right\|_{L^{1}(E)} \leq D(\varepsilon, t)\|f\|_{L^{1}(\mathfrak{X})},
$$

where

$$
D(\varepsilon, t)=\sup _{x \in \mathfrak{X}} \int_{\mathfrak{X} \backslash B(x, \varepsilon)} T_{t}(x, y) m(d y) \leq c_{4} \exp \left(-c_{5}\left(\varepsilon^{d_{w}} / t\right)^{1 /\left(d_{w}-1\right)}\right) .
$$

In particular, given any $s>0$ and $\varepsilon>0$ it is possible to choose a strictly increasing sequence $s_{j}>0$ convergent to $s$, with $s_{0}=0$, such that if $t_{j}=s_{j}-s_{j-1}(j \geq 1)$, then

$$
\lim _{j \rightarrow \infty} D\left(2^{-j} \varepsilon, s-s_{j}\right)=0 \quad \text { and } \quad \sum_{i=1}^{\infty} \frac{D\left(2^{-i} \varepsilon, t_{i}\right)}{t_{i+1}^{l}} \leq \frac{c_{6}\left(l, \varepsilon^{d_{w}} / s\right)}{\varepsilon^{l d_{w}}}<\infty
$$

for any $\varepsilon>0, l \geq 0$. For example, one can take $s_{j}=\left(1-4^{-d_{w} j}\right) s$. Note, however, that the above series would diverge if $t_{j}$ decreased either too slowly or too rapidly.

Step 2. Let $g \in L^{\infty}(\mathfrak{X}), \varepsilon, s>0$, and $h(x)=T_{s} g(x)$, as in the statement of the theorem. Following [71, for $j \geq 0$ we define

$$
K_{j}=\left\{x \in \mathfrak{X}: \operatorname{dist}(x, K)<2^{-j} \varepsilon\right\}, \quad L_{j}=\left\{x \in \mathfrak{X}: \operatorname{dist}(x, K)>\left(1-2^{-j}\right) \varepsilon\right\},
$$

and $A_{j}=\mathfrak{X} \backslash\left(K_{j} \cup L_{j}\right)$. Furthermore, let $s_{j}$ and $t_{j}$ be chosen as in Step 1. For $j \geq 1$ we define

$$
u_{0}(x)=0, \quad u_{j}(x)=\mathbf{1}_{K_{j}}(x) T_{s_{j}} g(x)+\mathbf{1}_{A_{j}}(x) T_{t_{j}} u_{j-1}(x) .
$$

Below we prove that $T_{s-s_{j}} u_{j}$ converges to a function $f$ with the desired properties.

Step 3. By induction, $\left\|u_{j}\right\|_{L^{\infty}(\mathfrak{X})} \leq\|g\|_{L^{\infty}(\mathfrak{X})}$ for any $j \geq 0$. For $j \geq 1$ we have $u_{j-1}(x)=T_{s_{j-1}} g(x)$ for $x \in K_{j-1}$, and $\operatorname{dist}\left(K_{j}, \mathfrak{X} \backslash K_{j-1}\right) \geq 2^{-j} \varepsilon$. Hence,

$$
\begin{aligned}
& \left\|u_{j}-T_{t_{j}} u_{j-1}\right\|_{L^{\infty}\left(K_{j}\right)}=\left\|T_{t_{j}}\left(T_{s_{j-1}} g-u_{j-1}\right)\right\|_{L^{\infty}\left(K_{j}\right)} \\
& \quad \leq D\left(2^{-j} \varepsilon, t_{j}\right)\left\|T_{s_{j-1}} g-u_{j-1}\right\|_{L^{\infty}(\mathfrak{X})} \leq 2 D\left(2^{-j} \varepsilon, t_{j}\right)\|g\|_{L^{\infty}(\mathfrak{X})},
\end{aligned}
$$

where $D\left(2^{-j} \varepsilon, t_{j}\right)$ is as in Step 1 (cf. [71, Lemma 2.3]). Also, $u_{j}$ vanishes on $L_{j}$, $u_{j-1}$ vanishes on $L_{j-1}$, and $\operatorname{dist}\left(L_{j}, \mathfrak{X} \backslash L_{j-1}\right) \geq 2^{-j} \varepsilon$, so that

$$
\left\|u_{j}-T_{t_{j}} u_{j-1}\right\|_{L^{\infty}\left(L_{j}\right)}=\left\|T_{t_{j}} u_{j-1}\right\|_{L^{\infty}\left(L_{j}\right)} \leq D\left(2^{-j} \varepsilon, t_{j}\right)\left\|u_{j-1}\right\|_{L^{\infty}(\mathfrak{X})},
$$

and (using $\mathfrak{X} \backslash L_{j-1} \subseteq K_{0}$ )

$$
\begin{aligned}
\left\|u_{j}-T_{t_{j}} u_{j-1}\right\|_{L^{1}\left(L_{j}\right)} & =\left\|T_{t_{j}} u_{j-1}\right\|_{L^{1}\left(L_{j}\right)} \\
& \leq D\left(2^{-j} \varepsilon, t_{j}\right)\left\|u_{j-1}\right\|_{L^{1}(\mathfrak{X})} \\
& \leq D\left(2^{-j} \varepsilon, t_{j}\right) m\left(K_{0}\right)\left\|u_{j-1}\right\|_{L^{\infty}(\mathfrak{X})} .
\end{aligned}
$$

Hence, using also $\left\|u_{j-1}\right\|_{L^{\infty}(\mathfrak{X})} \leq\|g\|_{L^{\infty}(\mathfrak{X})}$, we obtain (cf. [71, Lemma 2.5])

$$
\begin{aligned}
\left\|u_{j}-T_{t_{j}} u_{j-1}\right\|_{L^{2}\left(L_{j}\right)} & \leq \sqrt{\left\|u_{j}-T_{t_{j}} u_{j-1}\right\|_{L^{\infty}\left(L_{j}\right)}\left\|u_{j}-T_{t_{j}} u_{j-1}\right\|_{L^{1}\left(L_{j}\right)}} \\
& \leq D\left(2^{-j} \varepsilon, t_{j}\right) \sqrt{m\left(K_{0}\right)}\|g\|_{L^{\infty}(\mathfrak{X})} .
\end{aligned}
$$


Step 4. We follow the corrected version of the proof of [71, Lemma 2.6]. Let $l \geq 0$. For $j \geq 1$ we have

$$
\Delta^{l} T_{s-s_{j}} u_{j}=\sum_{i=1}^{j} \Delta^{l} T_{s-s_{i}}\left(u_{i}-T_{t_{i}} u_{i-1}\right) .
$$

Observe that the results of Step 1 and the equality $u_{i}(x)=T_{t_{i}} u_{i-1}(x)$ for $x \in A_{i}$ give

$$
\begin{gathered}
\sum_{i=1}^{\infty}\left\|\Delta^{l} T_{s-s_{i}}\left(u_{i}-T_{t_{i}} u_{i-1}\right)\right\|_{L^{\infty}(\mathfrak{X})} \leq \sum_{i=1}^{\infty} \frac{c_{3}(l)}{\left(s-s_{i}\right)^{l+n /\left(2 d_{w}\right)}}\left\|u_{i}-T_{t_{i}} u_{i-1}\right\|_{L^{2}(\mathfrak{X})} \\
\leq \sum_{i=1}^{\infty} \frac{c_{3}(l)}{t_{i+1}^{l+n /\left(2 d_{w}\right)}}\left(\left\|u_{i}-T_{t_{i}} u_{i-1}\right\|_{L^{2}\left(K_{i}\right)}+\left\|u_{i}-T_{t_{i}} u_{i-1}\right\|_{L^{2}\left(L_{i}\right)}\right) .
\end{gathered}
$$

Hence, by (A.2) and (A.3),

$$
\begin{aligned}
\sum_{i=1}^{\infty} \| & \Delta^{l} T_{s-s_{i}}\left(u_{i}-T_{t_{i}} u_{i-1}\right) \|_{L^{\infty}(\mathfrak{X})} \\
& \leq 3 c_{3}(l) \sqrt{m\left(K_{0}\right)}\|g\|_{L^{\infty}(\mathfrak{X})} \sum_{i=1}^{\infty} \frac{D\left(2^{-i} \varepsilon, t_{i}\right)}{t_{i+1}^{l+n /\left(2 d_{w}\right)}} \\
& \leq 3 c_{3}(l) \sqrt{m\left(K_{0}\right)}\|g\|_{L^{\infty}(\mathfrak{X})} \frac{c_{6}\left(l+n /\left(2 d_{w}\right), \varepsilon^{d_{w}} / s\right)}{\varepsilon^{l d_{w}+n / 2}} .
\end{aligned}
$$

It follows that the sequence $\Delta^{l} T_{s-s_{j}} u_{j}$ converges in $L^{\infty}(\mathfrak{X})$ as $j \rightarrow \infty$ for every $l \geq 0$. Therefore, if $f(x)=\lim _{j \rightarrow \infty} T_{s-s_{j}} u_{j}(x)$, then for all $l \geq 0$ we have $f \in \mathcal{D}\left(\Delta^{l}\right)$ and

$$
\begin{aligned}
\left\|\Delta^{l} f\right\|_{L^{\infty}(\mathfrak{X})} & \leq \sum_{i=1}^{\infty}\left\|\Delta^{l} T_{s-s_{i}}\left(u_{i}-T_{t_{i}} u_{i-1}\right)\right\|_{L^{\infty}(\mathfrak{X})} \\
& \leq \frac{c_{7}\left(l, \varepsilon^{d_{w}} / s\right)}{\varepsilon^{l d_{w}+n / 2}} \sqrt{m\left(K_{0}\right)}\|g\|_{L^{\infty}(\mathfrak{X})},
\end{aligned}
$$

as desired.

Step 5. By the definition of $u_{j}$, for $j \geq 1$ we have

$$
\begin{aligned}
T_{s-s_{j}} u_{j} & =T_{s-s_{j}}\left(\mathbf{1}_{K_{j}} T_{s_{j}} g+\mathbf{1}_{A_{j}} T_{t_{j}} u_{j-1}\right) \\
& =T_{s} g+T_{s-s_{j}}\left(\mathbf{1}_{A_{j}} T_{t_{j}} u_{j-1}-\mathbf{1}_{\mathfrak{X} \backslash K_{j}} T_{s_{j}} g\right) .
\end{aligned}
$$

It follows that

$$
\begin{aligned}
\left\|T_{s-s_{j}} u_{j}-T_{s} g\right\|_{L^{\infty}(K)} & =\left\|T_{s-s_{j}}\left(\mathbf{1}_{A_{j}} T_{t_{j}} u_{j-1}-\mathbf{1}_{\mathfrak{X} \backslash K_{j}} T_{s_{j}} g\right)\right\|_{L^{\infty}(K)} \\
& \leq D\left(2^{-j} \varepsilon, s-s_{j}\right)\left\|\mathbf{1}_{A_{j}} T_{t_{j}} u_{j-1}-\mathbf{1}_{\mathfrak{X} \backslash K_{j}} T_{s_{j}} g\right\|_{L^{\infty}(\mathfrak{X})} \\
& \leq 2 D\left(2^{-j} \varepsilon, s-s_{j}\right)\|g\|_{L^{\infty}(\mathfrak{X})} .
\end{aligned}
$$

The right hand side converges to 0 as $j \rightarrow \infty$. Hence, $f(x)=T_{s} g(x)=h(x)$ for $x \in$ $K$. Furthermore, $\left\|u_{j}\right\|_{L^{\infty}(\mathfrak{X})} \leq\|g\|_{L^{\infty}(\mathfrak{X})}$, and therefore also $\|f\|_{L^{\infty}(\mathfrak{X})} \leq\|g\|_{L^{\infty}(\mathfrak{X})}$. Finally, if $g \geq 0$, then $u_{j} \geq 0$ for all $j \geq 1$, and so $f \geq 0$.

By choosing $g(x)=h(x)=1$ and $s=\varepsilon^{d_{w}}$, we obtain the following result. 
Corollary A.2. Suppose that $K \subseteq \mathfrak{X}$ is a compact set and $\varepsilon>0$. Then there is a function $f \in L^{\infty}(\mathfrak{X})$ such that $f(x)=1$ for $x \in K, f(x)=0$ when $\operatorname{dist}(x, K) \geq \varepsilon$, and $f \in \mathcal{D}\left(\Delta^{l}\right)$ for any $l>0$. Furthermore, $0 \leq f(x) \leq 1$ for all $x \in \mathfrak{X}$, and for all $l>0$ we have

$$
\left\|\Delta^{l} f\right\|_{L^{\infty}(\mathfrak{X})} \leq \frac{{ }^{c \mid \underline{\mathrm{A} .4})}(\operatorname{diam} K+\varepsilon)^{n / 2}}{\varepsilon^{l d_{w}+n / 2}},
$$

where $c_{\underline{\mathrm{A} .4}}=c_{\underline{\mathrm{A} .4}}\left(l, Z_{t}\right)$.

In general, the boundary of the set $\{x \in \mathfrak{X}: f(x)>0\}$ might be highly irregular. However, when we relax the smoothness hypothesis on $f$, we can require $f$ to be positive on an arbitrary given open set.

Proposition A.3. Suppose that $K \subseteq \mathfrak{X}$ is a compact set, $\varepsilon>0$ and $L>0$. Then there is a function $f \in L^{\infty}(\mathfrak{X})$ such that $f(x)=1$ for $x \in K, f(x)=0$ when $\operatorname{dist}(x, K) \geq \varepsilon$, and $f \in \mathcal{D}\left(\Delta^{l}\right)$ for $l=1,2, \ldots, L$. Furthermore, $0 \leq f(x) \leq 1$ for all $x \in \mathfrak{X}$, the boundary of the set $\{x \in \mathfrak{X}: f(x)>0\}$ has zero $m$ measure, and for all $l=1,2, \ldots, L$ we have

$$
\left\|\Delta^{l} f\right\|_{L^{\infty}(\mathfrak{X})} \leq \frac{c_{\text {A.5 }}(\operatorname{diam} K+\varepsilon)^{n / 2}}{\varepsilon^{l d_{w}+n / 2}},
$$

where $c_{\underline{\mathrm{A} .5}}=c_{\underline{\mathrm{A} .5 \mathrm{t}}}\left(L, Z_{t}\right)$.

Proof. Let $f_{0}$ be the function constructed in Theorem A.1 for $h(x)=g(x)=1$, and denote by $V$ an arbitrary open set with the following properties: $\{x \in \mathfrak{X}: f(x)>$ $0\} \subseteq V \subseteq\{x \in \mathfrak{X}: \operatorname{dist}(x, K)<2 \varepsilon\}$, and $m(\partial V)=0$. For example, one can take $V=\{x \in \mathfrak{X}: \operatorname{dist}(x, K)<r\}$ for a suitable $r \in(\varepsilon, 2 \varepsilon)$.

Let $B_{j}, j=1,2, \ldots$, be a family of balls contained in $V \cap\left\{x \in \mathfrak{X}: f_{0}(x)<1 / 2\right\}$ such that twice smaller balls $B_{j}^{\prime}$ form a countable covering of $V \cap\left\{x \in \mathfrak{X}: f_{0}(x)<\right.$ $1 / 2\}$, and let $f_{j}$ be the function as in Corollary A.2. equal to 1 on $B_{j}^{\prime}$ and vanishing on $\mathfrak{X} \backslash B_{j}$. Finally, choose $\varepsilon_{j}>0$ so that for $l=0,1, \ldots, L$,

$$
\sum_{i=1}^{\infty} \varepsilon_{i}\left\|\Delta^{l} f_{i}\right\|_{L^{\infty}(\mathfrak{X})}<\frac{1}{2}\left\|\Delta^{l} f_{0}\right\|_{L^{\infty}(\mathfrak{X})} .
$$

Then $f=f_{0}+\sum_{i=1}^{\infty} \varepsilon_{i} f_{i}$ has all the desired properties, with $\varepsilon$ replaced by $2 \varepsilon$.

Corollary A.4. Assumption $\mathrm{B}$ holds with $\alpha$-stable scaling.

Proof. Given any compact subset $K$ of an open set $D \subseteq \mathfrak{X}$, choose $\varepsilon>0$ such that $\operatorname{dist}(\mathfrak{X} \backslash D, K) \geq \varepsilon$. Since $\mathcal{D}(\Delta) \subseteq \mathcal{D}(\mathcal{A})$, the function $f$ given in Proposition A.3 (for $L=1$ ) satisfies all conditions of Assumption B. Furthermore, if $\nu_{\eta}(s) d s$ is the Lévy measure of the subordinator $\eta_{t}$, then

$$
\begin{aligned}
\|\mathcal{A} f\|_{L^{\infty}(\mathfrak{X})} & =\left\|\int_{0}^{\infty}\left(T_{s}^{Z} f-f\right) \nu_{\eta}(s) d s\right\|_{L^{\infty}(\mathfrak{X})} \leq \int_{0}^{\infty}\left\|T_{s}^{Z} f-f\right\|_{L^{\infty}(\mathfrak{X})} \nu_{\eta}(s) d s \\
& \leq \int_{0}^{\infty} \min \left(s\|\Delta f\|_{L^{\infty}(\mathfrak{X})}, 2\|f\|_{L^{\infty}(\mathfrak{X})}\right) \nu_{\eta}(s) d s .
\end{aligned}
$$

Note that $\|f\|_{L^{\infty}(\mathfrak{X})}=1$. Furthermore, $\min (\lambda s, 2) \leq c_{1}\left(1-e^{-\lambda s}\right)$ (with $c_{1}=$ $\left.2 e^{2} /\left(e^{2}-1\right)\right)$, and

$$
\int_{0}^{\infty}\left(1-e^{-\lambda s}\right) \nu_{\eta}(s) d s=\lambda^{\alpha / d_{w}}
$$


Therefore,

$$
\|\mathcal{A} f\|_{L^{\infty}(\mathfrak{X})} \leq c_{1}\left(\|\Delta f\|_{L^{\infty}(\mathfrak{X})}\right)^{\alpha / d_{w}} .
$$

Let $0<r<R$, and take $K=\bar{B}\left(x_{0}, r\right), D=B\left(x_{0}, R\right), \varepsilon=R-r$. We see that

$$
\|\mathcal{A} f\|_{L^{\infty}(\mathfrak{X})} \leq c_{1}\left(\frac{c_{\left[\frac{\mathrm{A} .55}{}\right.}\left(1, Z_{t}\right)(2 R)^{n / 2}}{(R-r)^{d_{w}+n / 2}}\right)^{\alpha / d_{w}}=c_{2}\left(r / R, Z_{t}\right) R^{-\alpha} .
$$

This gives half of the $\alpha$-stable scaling property (f) and the other half is proved in a similar manner.

\section{ACKNOWLEDGMENTS}

The authors express their gratitude to Moritz Kaßmann for many discussions on the subject of supremum bounds for subharmonic functions. The authors thank Tomasz Grzywny for pointing out errors in the preliminary version of this article. The authors also thank Luke Rogers for providing them with a new proof of Theorem 2.2 in [71]. The authors thank the referee for insightful suggestions.

\section{REFERENCES}

[1] Hiroaki Aikawa, Boundary Harnack principle and Martin boundary for a uniform domain, J. Math. Soc. Japan 53 (2001), no. 1, 119-145, DOI 10.2969/jmsj/05310119. MR.1800526 (2001m:31007)

[2] Hiroaki Aikawa, Equivalence between the boundary Harnack principle and the Carleson estimate, Math. Scand. 103 (2008), no. 1, 61-76. MR2464701 (2009j:31008)

[3] Hiroaki Aikawa, Boundary Harnack principle and the quasihyperbolic boundary condition, Sobolev spaces in mathematics. II, Int. Math. Ser. (N. Y.), vol. 9, Springer, New York, 2009, pp. 19-30, DOI 10.1007/978-0-387-85650-6_3. MR2484620 (2010b:31009)

[4] Alano Ancona, Principe de Harnack à la frontière et théorème de Fatou pour un opérateur elliptique dans un domaine lipschitzien (French, with English summary), Ann. Inst. Fourier (Grenoble) 28 (1978), no. 4, 169-213, x. MR513885(80d:31006)

[5] Rodrigo Bañuelos and Krzysztof Bogdan, Symmetric stable processes in cones, Potential Anal. 21 (2004), no. 3, 263-288, DOI 10.1023/B:POTA.0000033333.72236.dc. MR2075671 (2005h:60238)

[6] Guy Barles, Emmanuel Chasseigne, and Cyril Imbert, Hölder continuity of solutions of second-order non-linear elliptic integro-differential equations, J. Eur. Math. Soc. (JEMS) 13 (2011), no. 1, 1-26, DOI 10.4171/JEMS/242. MR2735074(2011j:45008)

[7] Martin T. Barlow and Richard F. Bass, Transition densities for Brownian motion on the Sierpiński carpet, Probab. Theory Related Fields 91 (1992), no. 3-4, 307-330, DOI 10.1007/BF01192060. MR1151799 (93k:60203)

[8] Martin T. Barlow and Richard F. Bass, Brownian motion and harmonic analysis on Sierpiński carpets, Canad. J. Math. 51 (1999), no. 4, 673-744, DOI 10.4153/CJM-1999-031-4. MR.1701339 (2000i:60083)

[9] Martin T. Barlow, Richard F. Bass, Zhen-Qing Chen, and Moritz Kassmann, Non-local Dirichlet forms and symmetric jump processes, Trans. Amer. Math. Soc. 361 (2009), no. 4, 1963-1999, DOI 10.1090/S0002-9947-08-04544-3. MR2465826 (2010e:60163)

[10] Martin T. Barlow, Alexander Grigor'yan, and Takashi Kumagai, Heat kernel upper bounds for jump processes and the first exit time, J. Reine Angew. Math. 626 (2009), 135-157, DOI 10.1515/CRELLE.2009.005. MR2492992 (2009m:58077)

[11] Martin T. Barlow and Edwin A. Perkins, Brownian motion on the Sierpinski gasket, Probab. Theory Related Fields 79 (1988), no. 4, 543-623, DOI 10.1007/BF00318785. MR.966175 (89g:60241)

[12] Richard F. Bass, Adding and subtracting jumps from Markov processes, Trans. Amer. Math. Soc. 255 (1979), 363-376, DOI 10.2307/1998181. MR542886 (81b:60070) 
[13] Richard F. Bass and Krzysztof Burdzy, A probabilistic proof of the boundary Harnack principle, Seminar on Stochastic Processes, 1989 (San Diego, CA, 1989), Progr. Probab., vol. 18, Birkhäuser Boston, Boston, MA, 1990, pp. 1-16. MR1042338 (92c:60106)

[14] Richard F. Bass and Krzysztof Burdzy, A boundary Harnack principle in twisted Hölder domains, Ann. of Math. (2) 134 (1991), no. 2, 253-276, DOI 10.2307/2944347. MR 1127476 (92m:31006)

[15] Richard F. Bass and Krzysztof Burdzy, The boundary Harnack principle for nondivergence form elliptic operators, J. London Math. Soc. (2) 50 (1994), no. 1, 157-169, DOI 10.1112/jlms/50.1.157. MR.1277760(95f:35058)

[16] Richard F. Bass and David A. Levin, Harnack inequalities for jump processes, Potential Anal. 17 (2002), no. 4, 375-388, DOI 10.1023/A:1016378210944. MR1918242(2003e:60194)

[17] Albert Benveniste and Jean Jacod, Systèmes de Lévy des processus de Markov (French), Invent. Math. 21 (1973), 183-198. MR0343375 (49 \#8117)

[18] Robert M. Blumenthal and Ronald K. Getoor, Markov processes and potential theory, Pure and Applied Mathematics, Vol. 29, Academic Press, New York, 1968. MR0264757(41 \#9348)

[19] Krzysztof Bogdan, The boundary Harnack principle for the fractional Laplacian, Studia Math. 123 (1997), no. 1, 43-80. MR1438304 (98g:31005)

[20] Krzysztof Bogdan, Sharp estimates for the Green function in Lipschitz domains, J. Math. Anal. Appl. 243 (2000), no. 2, 326-337, DOI 10.1006/jmaa.1999.6673. MR.1741527 (2001b:31007)

[21] Krzysztof Bogdan, Krzysztof Burdzy, and Zhen-Qing Chen, Censored stable processes, Probab. Theory Related Fields 127 (2003), no. 1, 89-152, DOI 10.1007/s00440-003-02751. MR2006232 (2004g:60068)

[22] Krzysztof Bogdan and Tomasz Byczkowski, Potential theory for the $\alpha$-stable Schrödinger operator on bounded Lipschitz domains, Studia Math. 133 (1999), no. 1, 53-92. MR.1671973 (99m:31010)

[23] Krzysztof Bogdan, Tomasz Byczkowski, Tadeusz Kulczycki, Michal Ryznar, RenMing Song, and Zoran Vondraček, Potential analysis of stable processes and its extensions, Lecture Notes in Mathematics, vol. 1980, Springer-Verlag, Berlin, 2009. Edited by Piotr Graczyk and Andrzej Stos. MR2569321 (2011i:60140)

[24] Krzysztof Bogdan, Tomasz Grzywny, and Michał Ryznar, Heat kernel estimates for the fractional Laplacian with Dirichlet conditions, Ann. Probab. 38 (2010), no. 5, 1901-1923, DOI 10.1214/10-AOP532. MR2722789(2011f:60151)

[25] Krzysztof Bogdan and Tomasz Jakubowski, Estimates of the Green function for the fractional Laplacian perturbed by gradient, Potential Analysis 36 (2012), no. 3, 455-481. MR2892584

[26] Krzysztof Bogdan, Tadeusz Kulczycki, and Mateusz Kwaśnicki, Estimates and structure of $\alpha$-harmonic functions, Probab. Theory Related Fields 140 (2008), no. 3-4, 345-381, DOI 10.1007/s00440-007-0067-0. MR2365478 (2008k:31019)

[27] Krzysztof Bogdan, Andrzej Stós, and Paweł Sztonyk, Harnack inequality for stable processes on d-sets, Studia Math. 158 (2003), no. 2, 163-198, DOI 10.4064/sm158-2-5. MR2013738 (2005i:60092)

[28] Krzysztof Bogdan and Paweł Sztonyk, Estimates of the potential kernel and Harnack's inequality for the anisotropic fractional Laplacian, Studia Math. 181 (2007), no. 2, 101-123, DOI 10.4064/sm181-2-1. MR2320691(2008k:31015)

[29] Zhen-Qing Chen and Panki Kim, Green function estimate for censored stable processes, Probab. Theory Related Fields 124 (2002), no. 4, 595-610, DOI 10.1007/s00440-002-0226-2. MR1942325(2003m:60212)

[30] Zhen-Qing Chen, Panki Kim, and Takashi Kumagai, Weighted Poincaré inequality and heat kernel estimates for finite range jump processes, Math. Ann. 342 (2008), no. 4, 833-883, DOI 10.1007/s00208-008-0258-8. MR2443765 (2010b:60230)

[31] Zhen-Qing Chen, Panki Kim, and RenMing Song, Heat kernel estimates for the Dirichlet fractional Laplacian, J. Eur. Math. Soc. (JEMS) 12 (2010), no. 5, 1307-1329, DOI 10.4171/JEMS/231. MR2677618(2012c:58058)

[32] Zhen-Qing Chen, Panki Kim, RenMing Song, and Zoran Vondraček, Boundary Harnack principle for $\Delta+\Delta^{\alpha / 2}$, Trans. Amer. Math. Soc. 364 (2012), no. 8, 4169-4205, DOI 10.1090/S0002-9947-2012-05542-5. MR2912450 
[33] Zhen-Qing Chen and Takashi Kumagai, Heat kernel estimates for stable-like processes on dsets, Stochastic Process. Appl. 108 (2003), no. 1, 27-62, DOI 10.1016/S0304-4149(03)00105-4. MR2008600 (2005d:60135)

[34] Zhen-Qing Chen and Takashi Kumagai, A priori Hölder estimate, parabolic Harnack principle and heat kernel estimates for diffusions with jumps, Rev. Mat. Iberoam. 26 (2010), no. 2, 551-589, DOI 10.4171/RMI/609. MR2677007 (2011h:47093)

[35] Zhen-Qing Chen and Kazuhiro Kuwae, On subhamonicity for symmetric Markov processes, J. Math. Soc. Japan. 64 (2012), no. 4, 1181-1209. MR.2998921

[36] Zhen-Qing Chen and RenMing Song, Estimates on Green functions and Poisson kernels for symmetric stable processes, Math. Ann. 312 (1998), no. 3, 465-501, DOI 10.1007/s002080050232. MR.1654824 (2000b:60179)

[37] Kai Lai Chung and John B. Walsh, Markov processes, Brownian motion, and time symmetry, 2nd ed., Grundlehren der Mathematischen Wissenschaften [Fundamental Principles of Mathematical Sciences], vol. 249, Springer, New York, 2005. MR2152573 (2006j:60003)

[38] Björn E. J. Dahlberg, Estimates of harmonic measure, Arch. Rational Mech. Anal. 65 (1977), no. 3, 275-288. MR0466593 (57 \#6470)

[39] Claude Dellacherie and Paul-André Meyer, Probabilities and potential. C, North-Holland Mathematics Studies, vol. 151, North-Holland Publishing Co., Amsterdam, 1988. Potential theory for discrete and continuous semigroups; Translated from the French by J. Norris. MR.939365 (89b:60002)

[40] Eugene B. Dynkin, Markov processes. Vols. I, II, Translated with the authorization and assistance of the author by J. Fabius, V. Greenberg, A. Maitra, G. Majone. Die Grundlehren der Mathematischen Wissenschaften, Bände 121, vol. 122, Academic Press Inc., Publishers, New York, 1965. MR.0193671(33 \#1887)

[41] Pat J. Fitzsimmons, Ben M. Hambly, and Takashi Kumagai, Transition density estimates for Brownian motion on affine nested fractals, Comm. Math. Phys. 165 (1994), no. 3, 595-620. MR.1301625 (95j:60122)

[42] David Gilbarg and Neil S. Trudinger, Elliptic partial differential equations of second order, 2nd ed., Grundlehren der Mathematischen Wissenschaften [Fundamental Principles of Mathematical Sciences], vol. 224, Springer-Verlag, Berlin, 1983. MR737190 (86c:35035)

[43] Alexander Grigor'yan, Heat kernel and analysis on manifolds, AMS/IP Studies in Advanced Mathematics, vol. 47, American Mathematical Society, Providence, RI, 2009. MR2569498 (2011e:58041)

[44] Qing-Yang Guan, Boundary Harnack inequalities for regional fractional Laplacian. ArXiv e-prints, May 2007.

[45] Qing-Yang Guan and Zhi-Ming Ma, Reflected symmetric $\alpha$-stable processes and regional fractional Laplacian, Probab. Theory Related Fields 134 (2006), no. 4, 649-694, DOI 10.1007/s00440-005-0438-3. MR2214908 (2007a:60034)

[46] Pavel Gyrya and Laurent Saloff-Coste, Neumann and Dirichlet heat kernels in inner uniform domains (English, with English and French summaries), Astérisque 336 (2011), viii+144. MR:2807275(2012m:35117)

[47] Wolfhard Hansen, Uniform boundary Harnack principle and generalized triangle property, J. Funct. Anal. 226 (2005), no. 2, 452-484, DOI 10.1016/j.jfa.2004.12.010. MR2160104 (2006i:35004)

[48] Wolfhard Hansen, Three views on potential theory. Lecture notes for a course given at Charles University (Prague) in 2008. Available at http://www .karlin.mff . cuni.cz/ hansen/ lecture/course-07012009.pdf, 2009.

[49] Walter Hoh, Pseudo differential operators generating Markov processes, Habilitation, Universität Bielefeld, 1998. Available at http://www.mathematik.uni-bielefeld.de/ hoh/pdo_ $\mathrm{mp} \cdot \mathrm{ps}$.

[50] Walter Hoh, A symbolic calculus for pseudo-differential operators generating Feller semigroups, Osaka J. Math. 35 (1998), no. 4, 789-820. MR.1659620(99k:47102)

[51] Nobuyuki Ikeda and Shinzo Watanabe, On some relations between the harmonic measure and the Lévy measure for a certain class of Markov processes, J. Math. Kyoto Univ. 2 (1962), 79-95. MR0142153(25 \#5546)

[52] Tomasz Jakubowski, The estimates for the Green function in Lipschitz domains for the symmetric stable processes, Probab. Math. Statist. 22 (2002), no. 2, Acta Univ. Wratislav. No. 2470, 419-441. MR1991120 (2004g:60069) 
[53] Tomasz Jakubowski, Fractional Laplacian with singular drift, Studia Math. 207 (2011), no. 3, 257-273, DOI 10.4064/sm207-3-3. MR2875353(2012k:60226)

[54] David S. Jerison and Carlos E. Kenig, Boundary value problems on Lipschitz domains, Studies in partial differential equations, MAA Stud. Math., vol. 23, Math. Assoc. America, Washington, DC, 1982, pp. 1-68. MR 716504 (85f:35057)

[55] Kamil Kaleta and Mateusz Kwaśnicki, Boundary Harnack inequality for $\alpha$-harmonic functions on the Sierpiński triangle, Probab. Math. Statist. 30 (2010), no. 2, 353-368. MR2792590 (2012f:60271)

[56] Moritz Kassmann, A priori estimates for integro-differential operators with measurable kernels, Calc. Var. Partial Differential Equations 34 (2009), no. 1, 1-21, DOI 10.1007/s00526008-0173-6. MR2448308 (2010b:35474)

[57] John T. Kemper, A boundary Harnack principle for Lipschitz domains and the principle of positive singularities, Comm. Pure Appl. Math. 25 (1972), 247-255. MR0293114(45 \#2193)

[58] John T. Kemper, A correction to: "A boundary Harnack principle for Lipschitz domains and the principle of positive singularities" (Comm. Pure Appl. Math. 25 (1972), 247-255). Comm. Pure Appl. Math. 29 (1976), no. 5, 555. MR0422644 (54:10630)

[59] Jun Kigami, Analysis on fractals, Cambridge Tracts in Mathematics, vol. 143, Cambridge University Press, Cambridge, 2001. MR1840042 (2002c:28015)

[60] Panki Kim and Ante Mimica, Harnack inequalities for subordinate Brownian motions, Electron. J. Probab. 17 (2012), no. 37, 23. MR2928720

[61] Panki Kim and RenMing Song, Potential theory of truncated stable processes, Math. Z. 256 (2007), no. 1, 139-173, DOI 10.1007/s00209-006-0063-6. MR2282263 (2007k:60245)

[62] Panki Kim and RenMing Song, Boundary behavior of harmonic functions for truncated stable processes, J. Theoret. Probab. 21 (2008), no. 2, 287-321, DOI 10.1007/s10959-008-0145-y. MR2391246 (2010f:60230)

[63] Panki Kim, RenMing Song, and Zoran Vondraček, Boundary Harnack principle for subordinate Brownian motions, Stochastic Process. Appl. 119 (2009), no. 5, 1601-1631, DOI 10.1016/j.spa.2008.08.003. MR2513121(2010f:60231)

[64] Panki Kim, RenMing Song, and Zoran Vondraček, Potential theory of subordinate Brownian motions revisited, Stochastic analysis and applications to finance, Interdiscip. Math. Sci., vol. 13, World Sci. Publ., Hackensack, NJ, 2012, pp. 243-290. MR2986850

[65] Panki Kim, RenMing Song, and Zoran Vondraček, Uniform boundary Harnack principle for rotationally symmetric Lévy processes in general open sets, Sci. China Math. 55 (2012), no. 11, 2317-2333, DOI 10.1007/s11425-012-4516-6. MR2994122

[66] Panki Kim, RenMing Song, and Zoran Vondraček, Two-sided Green function estimates for killed subordinate Brownian motions, Proc. Lond. Math. Soc. (3) 104 (2012), no. 5, 927-958. MR2928332

[67] Tadeusz Kulczycki, Properties of Green function of symmetric stable processes, Probab. Math. Statist. 17 (1997), no. 2, Acta Univ. Wratislav. No. 2029, 339-364. MR.1490808 (98m:60119)

[68] Takashi Kumagai, Estimates of transition densities for Brownian motion on nested fractals, Probab. Theory Related Fields 96 (1993), no. 2, 205-224, DOI 10.1007/BF01192133. MR.1227032(94e:60068)

[69] Takashi Kumagai, Some remarks for stable-like jump processes on fractals, Fractals in Graz 2001, Trends Math., Birkhäuser, Basel, 2003, pp. 185-196. MR2091704 (2006a:60138)

[70] P. Joseph McKenna and Wolfgang Reichel, A priori bounds for semilinear equations and a new class of critical exponents for Lipschitz domains, J. Funct. Anal. 244 (2007), no. 1, 220-246, DOI 10.1016/j.jfa.2006.11.018. MR2294482 (2007k:35158)

[71] Luke G. Rogers, Robert S. Strichartz, and Alexander Teplyaev, Smooth bumps, a Borel theorem and partitions of smooth functions on P.C.F. fractals, Trans. Amer. Math. Soc. 361 (2009), no. 4, 1765-1790, DOI 10.1090/S0002-9947-08-04772-7. MR2465816 (2010f:28020)

[72] Ken-iti Sato, Lévy processes and infinitely divisible distributions, Cambridge Studies in Advanced Mathematics, vol. 68, Cambridge University Press, Cambridge, 1999. Translated from the 1990 Japanese original; Revised by the author. MR.1739520 (2003b:60064)

[73] René L. Schilling, RenMing Song, and Zoran Vondraček, Bernstein functions, de Gruyter Studies in Mathematics, vol. 37, Walter de Gruyter \& Co., Berlin, 2010. Theory and applications. MR2598208(2011d:60060) 
[74] Michael Sharpe, General theory of Markov processes, Pure and Applied Mathematics, vol. 133, Academic Press Inc., Boston, MA, 1988. MR958914 (89m:60169)

[75] RenMing Song and Jang-Mei Wu, Boundary Harnack principle for symmetric stable processes, J. Funct. Anal. 168 (1999), no. 2, 403-427, DOI 10.1006/jfan.1999.3470. MR1719233 (2001b:60092)

[76] Andrzej Stós, Symmetric $\alpha$-stable processes on d-sets, Bull. Polish Acad. Sci. Math. 48 (2000), no. 3, 237-245. MR.1779007 (2002f:60152)

[77] Andrzej Stós, Boundary Harnack principle for fractional powers of Laplacian on the Sierpiński carpet (English, with English and French summaries), Bull. Sci. Math. 130 (2006), no. 7, 580-594, DOI 10.1016/j.bulsci.2006.01.001. MR.2261965(2008h:60320)

[78] Robert S. Strichartz and Michael Usher, Splines on fractals, Math. Proc. Cambridge Philos. Soc. 129 (2000), no. 2, 331-360, DOI 10.1017/S0305004100004424. MR1765920 (2001c:28016)

[79] Paweł Sztonyk, Approximation of stable-dominated semigroups, Potential Anal. 33 (2010), no. 3, 211-226, DOI 10.1007/s11118-009-9165-1. MR2718253 (2011i:60158)

[80] Shinzo Watanabe, On discontinuous additive functionals and Lévy measures of a Markov process, Japan. J. Math. 34 (1964), 53-70. MR0185675 (32 \#3137)

[81] Takesi Watanabe, On the equivalence of excessive functions and superharmonic functions in the theory of Markov processes. I, Proc. Japan Acad. 38 (1962), 397-401. MR0150831 (27 \#818a)

[82] Toshiro Watanabe, Asymptotic estimates of multi-dimensional stable densities and their applications, Trans. Amer. Math. Soc. 359 (2007), no. 6, 2851-2879 (electronic), DOI 10.1090/S0002-9947-07-04152-9. MR2286060 (2008e:60143)

[83] Jang Mei G. Wu, Comparisons of kernel functions, boundary Harnack principle and relative Fatou theorem on Lipschitz domains (English, with French summary), Ann. Inst. Fourier (Grenoble) 28 (1978), no. 4, 147-167, vi. MR513884 (80g:31005)

[84] Zhong Xin Zhao, Green function for Schrödinger operator and conditioned Feynman-Kac gauge, J. Math. Anal. Appl. 116 (1986), no. 2, 309-334, DOI 10.1016/S0022-247X(86)800014. MR842803 (88f:60142)

Institute of Mathematics, Polish Academy of Science, ul. Śniadeckich 8, 00-958 Warsaw, Poland - And - Institute of Mathematics and Computer Science, WrocŁaW University of Technology, ul. Wybrzeże Wyspiańskiego 27, 50-370 WrocŁaW, Poland

E-mail address: krzysztof.bogdan@pwr.wroc.pl

Research Institute for Mathematical Sciences, Kyoto University, Kyoto 606-8502, JAPAN

E-mail address: kumagai@kurims.kyoto-u.ac.jp

Institute of Mathematics, Polish ACademy of Science, ul. Śniadeckich 8, 00-958 Warsaw, Poland - and - Institute of Mathematics and Computer Science, Wrocław University of Technology, ul. Wybrzeże Wyspiańskiego 27, 50-370 Wroceaw, Poland

E-mail address: mateusz.kwasnicki@pwr.wroc.pl 